\title{
Divalent Activation in Temporary Phosphate Tethers: Highly Selective Cuprate Displacement Reactions
}

Alan Whitehead, James P. McParland, and Paul R. Hanson*

Department of Chemistry, University of Kansas, 1251 Wescoe Hall Drive, Lawrence, KS 66045-7582

phanson@ku.edu

\section{Table of Contents}

General Experimental Methods:

Experimental Section and Characterization Data

S 3- S 15

NMR Spectra for New Compounds

S 16- S 43 


\section{General Experimental Methods.}

All air and moisture sensitive reactions were carried out in flame- or oven-dried glassware under argon atmosphere using standard gastight syringes, cannulaes, and septa. Stirring was achieved with oven-dried magnetic stir bars. $\mathrm{Et}_{2} \mathrm{O}$, toluene, THF and $\mathrm{CH}_{2} \mathrm{Cl}_{2}$ were purified by passage through the Solv-Tek purification system employing activated $\mathrm{Al}_{2} \mathrm{O}_{3}$ (Grubbs, R.H.; Rosen, R.K.; Timmers, F.J.; Organometallics 1996, 15, 1518-1520). $\mathrm{Et}_{3} \mathrm{~N}$ was purified by passage over basic alumina and stored over $\mathrm{KOH}$. Butyl Lithium was purchased from Aldrich and titrated prior to use. Dialkyl zinc and alkyl zinc halide reagents were purchased from Aldrich and were used at the reported molarities. Grubb's second-generation olefin metathesis catalyst was acquired from Materia and used without further purification. Flash column chromatography was performed with Merck silica gel (EM-9385-9, 230-400 mesh). Thin layer chromatography was performed on silica gel $60 \mathrm{~F}_{254}$ plates (EM-5717, Merck). Deuterated solvents were purchased from Cambridge Isotope laboratories. ${ }^{1} \mathrm{H},{ }^{13} \mathrm{C}$ and ${ }^{31} \mathrm{P}$ NMR spectra were recorded on a Bruker DRX400 spectrometer operating at $400 \mathrm{MHz}, 100 \mathrm{MHz}$, and $162 \mathrm{MHz}$ respectively or a Bruker Avance operating at $500 \mathrm{MHz}$ and $125 \mathrm{MHz}$ respectively. High-resolution mass spectrometry (HRMS) and FAB spectra were obtained on a VG Instrument ZAB double-focusing mass spectrometer. 
Experimental procedures and spectral data for compounds 1, 4-6, 8a, 9a-9h, 10a, 11, 12, 14, 15 and syn- and anti-ethyl-substituted acetonides.

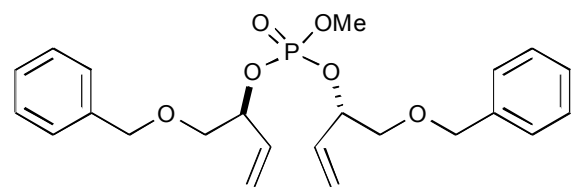

$(\boldsymbol{S}, \boldsymbol{S})$-acyclic diene phosphate triester: A solution of allylic alcohol $3(2.05 \mathrm{~g}, 11.54 \mathrm{mmol})$ in THF $(58 \mathrm{~mL})$ was cooled to $-30{ }^{\circ} \mathrm{C}$. BuLi $(2.47 \mathrm{M}, 11.02 \mathrm{mmol})$ was slowly added, followed by ten minutes of stirring. A solution of dichloromethyl phosphate $(781 \mathrm{mg}, 5.25 \mathrm{mmol})$ in THF $(10.5 \mathrm{~mL})$ was slowly cannulated into the reaction vessel containing the alkoxide. The reaction continued to stir at $-30{ }^{\circ} \mathrm{C}$ for 30 minutes, the cold bath was removed and the solution was warmed to room temperature and stirred for an additional two hours. Upon completion (monitored by TLC), the reaction was quenched with $20 \mathrm{~mL}$ of $\mathrm{NH}_{4} \mathrm{Cl}$ (sat'd aq). The separated aqueous layer was extracted with $\mathrm{Et}_{2} \mathrm{O}(3 \mathrm{x})$ and the combined organic layers washed with brine then dried $\left(\mathrm{MgSO}_{4}\right)$. Flash chromatography $(2: 1$ Hexanes/EtOAc) provided $1.96 \mathrm{~g}$ (87\% yield) of the diene product as a faint yellow oil. $[\alpha]_{\mathrm{D}}+0.54\left(c=0.37, \mathrm{CH}_{2} \mathrm{Cl}_{2}\right)$; IR (neat) 2930, 1452, 1266, 1016, 734, $698 \mathrm{~cm}^{-1} ;{ }^{1} \mathrm{H}$ NMR (500 MHz, $\left.\mathrm{CDCl}_{3}\right) \delta$ 7.4-7.3 (m, 10H), 5.91 (ddd, $J=17.2$, 10.6, $6.4 \mathrm{~Hz}, 1 \mathrm{H}), 5.91$ (ddd, $J=17.2,10.6,6.4 \mathrm{~Hz}, 1 \mathrm{H}), 5.45$ (d, $J=17.2,1 \mathrm{H}), 5.39$ (d, $J=$ $17.2,1 \mathrm{H}), 5.30(\mathrm{~d}, J=10.5,1 \mathrm{H}), 5.30(\mathrm{~d}, J=10.5,1 \mathrm{H}), 4.98-5.09(\mathrm{~m}, 2 \mathrm{H}), 4.59$ (dd, $J=12.2$, $5.1 \mathrm{~Hz}, 2 \mathrm{H}), 4.54(\mathrm{dd}, J=12,1.4 \mathrm{~Hz}, 2 \mathrm{H}), 3.72(\mathrm{~d}, J=11.4 \mathrm{~Hz}, 3 \mathrm{H}), 3.62(\mathrm{dd}, J=10.6,6.3 \mathrm{~Hz}$, 2H), $3.57(\mathrm{~m}, 2 \mathrm{H}) ;{ }^{13} \mathrm{C} \mathrm{NMR}\left(125 \mathrm{MHz}, \mathrm{CDCl}_{3}\right) \delta 138.11,134.05,134.02,133.99,128.56$, 127.89, 118.84, 118.65, 78.13, 78.10, 78.09, 78.05, 73.44, 73.41, 72.47(d, $\left.J_{\mathrm{CP}}=0.8 \mathrm{~Hz}\right), 72.42$ $\left(\mathrm{d}, J_{\mathrm{CP}}=1.5 \mathrm{~Hz}\right), 54.48,54.44 ;{ }^{31} \mathrm{P} \mathrm{NMR}\left(162 \mathrm{MHz}, \mathrm{CDCl}_{3}\right) \delta 0.29 ;$ HRMS calculated for $\mathrm{C}_{23} \mathrm{H}_{29} \mathrm{O}_{6} \mathrm{P}(\mathrm{M}+\mathrm{H})^{+} 433.1780$; found 433.1757 (FAB).

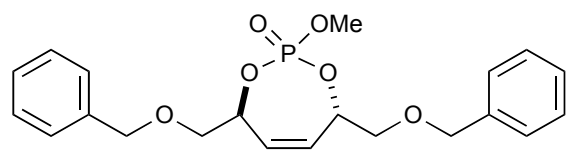

$(S, S)$-monocyclic phosphate 1: A flask containing the $(S, S)$-diene phosphate triester (1.45 g, $3.36 \mathrm{mmol})$ was charged with toluene $(670 \mathrm{~mL})$ that had been degassed for 20 minutes with argon. The solution was brought to reflux while continually sparging with argon, and $\left(\mathrm{ImesH}_{2}\right)\left(\mathrm{PCy}_{3}\right)\left(\mathrm{Cl}_{2}\right) \mathrm{Ru}=\mathrm{CHPh}$ was added $(200 \mathrm{mg}, 0.235 \mathrm{mmol})$. The reaction was maintained at reflux under argon sparging for 30 minutes. Upon completion (monitored by TLC) the reaction was cooled to room temperature and concentrated under reduced pressure. Purification 
via flash chromatography (1:1 Hexanes/EtOAc) supplied $927 \mathrm{mg}$ (68\% yield) of monocylic phosphate 1. $[\alpha]_{\mathrm{D}}-52.6\left(c=1.6, \mathrm{CH}_{2} \mathrm{Cl}_{2}\right)$; IR (neat) $3029,1454,1365,1282,1027 \mathrm{~cm}^{-1} ;{ }^{1} \mathrm{H}$ $\operatorname{NMR}\left(400 \mathrm{MHz}, \mathrm{CDCl}_{3}\right) \delta 7.28-7.39(\mathrm{~m}, 10 \mathrm{H}), 5.76(\mathrm{ddd}, J=12.0,1.8,1.6 \mathrm{~Hz}, 1 \mathrm{H}), 5.72$ (ddd, $J=12.0,1.8,1.6 \mathrm{~Hz}, 1 \mathrm{H}), 5.26-5.32(\mathrm{~m}, 1 \mathrm{H}), 5.07-5.14(\mathrm{~m}, 1 \mathrm{H}), 4.56-4.64(\mathrm{~m}, 4 \mathrm{H}), 3.87\left(\mathrm{~d}, J_{\mathrm{HP}}\right.$ $=11.1 \mathrm{~Hz}, 3 \mathrm{H}$ ), 3.67 (dddd, $J=6.4,5.5,5.1,0.9 \mathrm{~Hz}, 2 \mathrm{H}$ ), 3.59 (dddd, $J=5.3,5.1,2.1,0.5 \mathrm{~Hz}$, $2 \mathrm{H}) ;{ }^{13} \mathrm{C}$ NMR $\left(100 \mathrm{MHz}, \mathrm{CDCl}_{3}\right) \delta 137.56,137.48,129.49,128.42,128.09,127.83,127.81$, $127.69,127.63,73.91,\left(\mathrm{~d}, J_{\mathrm{CP}}=7.0\right), 73.62\left(\mathrm{~d}, J_{\mathrm{CP}}=4.5\right), 73.49,73.33,71.60\left(\mathrm{~d}, J_{\mathrm{CP}}=11.9\right)$, $71.21\left(J_{\mathrm{CP}}=12.1\right), 54.62\left(\mathrm{~d}, J_{\mathrm{CP}}=5.1\right) ;{ }^{31} \mathrm{P}$ NMR $\left(162 \mathrm{MHz}, \mathrm{CDCl}_{3}\right) \delta 3.61$; HRMS calculated for $\mathrm{C}_{21} \mathrm{H}_{25} \mathrm{O}_{6} \mathrm{P}(\mathrm{M}+\mathrm{H})^{+}$405.1467; found $405.1437(\mathrm{FAB})$.

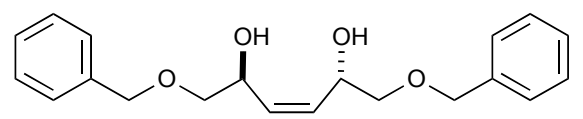

(2S, 5S, 3Z)-1,6-bis(benzyloxy)hex-3-ene-2,5-diol 4: Phosphate 1 (30 mg, $0.074 \mathrm{mmol}$ ) was taken up in THF $(0.20 \mathrm{~mL})$ and cooled to $0{ }^{\circ} \mathrm{C}$ while stirring under argon. $\mathrm{LiAlH}_{4}(0.010 \mathrm{mg}$, $0.24 \mathrm{mmol}$ ) was added slowly, and upon complete addition stirring continued for an additional 2 hours while warming to room temperature. The mixture was then cooled to $0{ }^{\circ} \mathrm{C}$ and slowly quenched with $\mathrm{H}_{2} \mathrm{O}(0.1 \mathrm{~mL})$ followed by $10 \% \mathrm{NaOH}$ aq $(0.2 \mathrm{~mL})$. The layers were separated and the aqueous layer extracted with EtOAc (5x). The combined organic layers were concentrated under reduced pressure and dried $\left(\mathrm{Na}_{2} \mathrm{SO}_{4}\right)$. Column chromatography (5:1 Heptane:EtOAc) provided $4(18.3 \mathrm{mg}, 75 \%)$ as a clear oil. $[\alpha]_{\mathrm{D}}+21.7\left(c=1.7, \mathrm{CH}_{2} \mathrm{Cl}_{2}\right)$; IR (neat) $3417,3025,2902,1493,1465,1101,1075,737,698 \mathrm{~cm}^{-1} ;{ }^{1} \mathrm{H}$ NMR $\left(400 \mathrm{MHz}, \mathrm{CDCl}_{3}\right) \delta$ 7.28-7.37 (m, 10H), 5.58-5.64 (m, 2H), 4.63-4.68 (m, 2H), 4.57 (s, 2H), 3.53 (dd, $J=9.4,4.4 \mathrm{~Hz}$ $2 \mathrm{H}), 3.46(\mathrm{dd}, J=9.5,7.4 \mathrm{~Hz}, 1 \mathrm{H}), 2.65(\mathrm{bs}, 2 \mathrm{H}) ;{ }^{13} \mathrm{C} \mathrm{NMR}\left(100 \mathrm{MHz}, \mathrm{CDCl}_{3}\right) \delta 137.62$, 132.08, 128.47, 127.87, 127.84, 73.48, 73.42, 67.21. HRMS calculated for $\mathrm{C}_{20} \mathrm{H}_{24} \mathrm{O}_{4}(\mathrm{M}+\mathrm{Na})^{+}$ 351.1572; found 351.1582 (FAB).

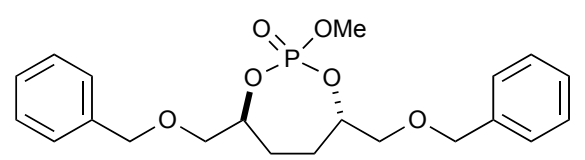

(S,S)-hydrogenated monocyclic phosphate 5: Post ring-closing metathesis (50 mg, 0.123 mmol) with $\left(\mathrm{ImesH}_{2}\right)\left(\mathrm{PCy}_{3}\right)\left(\mathrm{Cl}_{2}\right) \mathrm{Ru}=\mathrm{CHPh}(33 \mathrm{mg}, 0.040 \mathrm{mmol})$, the reaction was a concentrated under reduced pressure and the resulting brown oil was taken up in dry $\mathrm{CH}_{2} \mathrm{Cl}_{2}(10$ $\mathrm{mL}$ ). The reaction was cannulated to a hydrogen Parr bomb apparatus, followed by 
implementing three quick cycles of evacuation and hydrogen purging. The bomb was capped and charged with $\mathrm{H}_{2}$ (300 psi) while heating at $80{ }^{\circ} \mathrm{C}$. After stirring for two hours, the reaction mixture was concentrated under reduced pressure and immediately subjected to column chromatography (1:1 EtOAc:Heptane) providing $45.4 \mathrm{mg}$ of 5 (90 \% yield) as a light brown oil (small ruthenium contamination estimated to be $<1 \%)$. $[\alpha]_{\mathrm{D}}-2.15\left(c=0.33, \mathrm{CH}_{2} \mathrm{Cl}_{2}\right)$; IR (neat) 2917, 1279, 1098, 1000, 737, $698 \mathrm{~cm}^{-1}$; ${ }^{1} \mathrm{H}$ NMR (400 MHz, $\left.\mathrm{CDCl}_{3}\right) \delta$ 7.25-7.37 (m, 10H), 4.52$4.61(\mathrm{~m}, 5 \mathrm{H}), 4.31-4.35(\mathrm{~m}, 1 \mathrm{H}), 3.82\left(\mathrm{~d}, J_{\mathrm{HP}}=11.1 \mathrm{~Hz}, 3 \mathrm{H}\right), 3.43-3.65(\mathrm{~m}, 4 \mathrm{H}), 2.06(\mathrm{dd}, J=$ 11.9, $4.3 \mathrm{~Hz}, 1 \mathrm{H}), 1.98(\mathrm{dd}, J=10.6,3.8 \mathrm{~Hz}, 1 \mathrm{H}), 1.73-1.82(\mathrm{~m}, 2 \mathrm{H}) ;{ }^{13} \mathrm{C} \mathrm{NMR}(100 \mathrm{MHz}$, $\left.\mathrm{CDCl}_{3}\right) \delta 137.67\left(\mathrm{~d}, J_{\mathrm{CP}}=2.6 \mathrm{~Hz}\right), 128.35,127.72,127.65,127.55,77.94\left(\mathrm{~d}, J_{\mathrm{CP}}=6.4 \mathrm{~Hz}\right)$, $76.38\left(\mathrm{~d}, J_{\mathrm{CP}}=3.7 \mathrm{~Hz}\right), 73.32\left(\mathrm{~d}, J_{\mathrm{CP}}=10.6 \mathrm{~Hz}\right), 72.59\left(\mathrm{~d}, J_{\mathrm{CP}}=11.1 \mathrm{~Hz}\right), 72.08\left(\mathrm{~d}, J_{\mathrm{CP}}=11.1\right.$

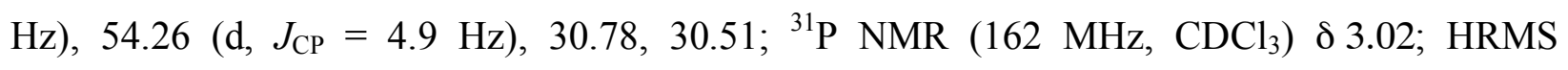
calculated for $\mathrm{C}_{21} \mathrm{H}_{27} \mathrm{O}_{6} \mathrm{P}(\mathrm{M}+\mathrm{H})^{+}$407.1624; found 407.1649 (FAB).

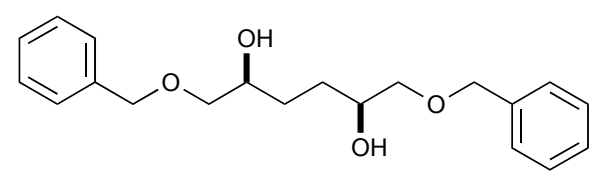

(2S, 5S)-1,6-bis(benzyloxy)hexane-2,5-diol 6: Phosphate 5 (30 mg, $0.074 \mathrm{mmol})$ was dissolved in THF $(1.2 \mathrm{~mL})$ and cooled to $0{ }^{\circ} \mathrm{C}$ while stirring under argon. $\mathrm{LiAlH}_{4}(0.0084 \mathrm{mg}, 0.22 \mathrm{mmol})$ was added slowly, and upon complete addition stirring continued for an additional 2 hours while warming to room temperature. An additional amount of $\mathrm{LiAlH}_{4}$ was added, and the solution stirred for an additional hour. The mixture was then cooled to $0{ }^{\circ} \mathrm{C}$ and slowly quenched with $\mathrm{H}_{2} \mathrm{O}(0.1 \mathrm{~mL})$ followed by $10 \% \mathrm{NaOH}$ aq $(0.2 \mathrm{~mL})$, and then $\mathrm{H}_{2} \mathrm{O}(0.1 \mathrm{~mL})$. The layers were separated and the aqueous layer extracted with EtOAc $(5 \mathrm{x})$. The combined organic layers were concentrated under reduced pressure and dried $\left(\mathrm{Na}_{2} \mathrm{SO}_{4}\right)$. Column chromatography (5:1 Heptane:EtOAc) provided $6(21.9 \mathrm{mg}, 90 \%)$ as a clear oil. $[\alpha]_{\mathrm{D}}+7.06\left(c=0.09, \mathrm{CH}_{2} \mathrm{Cl}_{2}\right) ; \mathrm{IR}$ (neat) 3436, 1096, 737, $698 \mathrm{~cm}^{-1}$; ${ }^{1} \mathrm{H}$ NMR $\left(500 \mathrm{MHz}, \mathrm{CDCl}_{3}\right) \delta 7.28-7.37(\mathrm{~m}, 10 \mathrm{H}), 4.55(\mathrm{~s}$, 2H), 3.82-3.88 (m, 2H), 3.49 (dd, $J=9.0,3.5 \mathrm{~Hz}, 2 \mathrm{H}), 3.49$ (dd, $J=9.0,7.5 \mathrm{~Hz}, 2 \mathrm{H}$ ), 2.25 (bs, 2H), 1.56-1.65 (m, 4H); ${ }^{13} \mathrm{C}$ NMR (125 MHz, $\left.\mathrm{CDCl}_{3}\right) \delta 137.93,128.46,127.79,127.75,74.41$, 73.34, 70.38, 29.53. HRMS calculated for $\mathrm{C}_{20} \mathrm{H}_{24} \mathrm{O}_{4}(\mathrm{M}+\mathrm{Na})^{+}$351.1572; found 351.1582 (FAB).

General Procedure A for Diakyl Zincate Derived Cuprates: To a solution of CuCN•2LiCl (1 mmol, $1 \mathrm{M}$ in THF) cooled to $-30{ }^{\circ} \mathrm{C}$ was added dropwise the dialkyl zinc reagent as a solution in 
THF ( $1 \mathrm{mmol}$ ). After stirring $30-45 \mathrm{~min}$ ( $\mathrm{Me}_{2} \mathrm{Zn}$ required longer stirring times) at $-25{ }^{\circ} \mathrm{C}$ to -30 ${ }^{\circ} \mathrm{C}$, a solution of phosphate $\mathbf{1}(0.20 \mathrm{mmol})$ in THF $(2 \mathrm{~mL})$ was added. The reaction progress was monitored by TLC (products are baseline in EtOAc), and upon completion where quenched with $10 \% \mathrm{HCl}$ aq. $(1 \mathrm{~mL})$ and stirred for $30-60 \mathrm{~min}$. The aqueous layer was extracted was extracted with $\mathrm{EtO}_{2}(4 \times 1 \mathrm{~mL})$ and the combined organic layers were subsequently washed with $\mathrm{H}_{2} \mathrm{O}(1 \mathrm{x}$ $1 \mathrm{~mL}$ ) and filtered of the resulting white solids. The aqueous layer was removed and the organic layer was concentrated under reduced pressure. The crude acids were used without further purification.

General Procedure B for Alkyl Zinc halide Derived Cuprates: To a solution of $\mathrm{CuCN} \cdot 2 \mathrm{LiCl}$ ( $1 \mathrm{mmol}, 1 \mathrm{M}$ in THF) cooled to $-30{ }^{\circ} \mathrm{C}$ was added dropwise the alkyl zinc halide reagent as a solution in THF $(1 \mathrm{mmol}, 1.0 \mathrm{M})$. After stirring for one hour at $-25{ }^{\circ} \mathrm{C}$ to $-30{ }^{\circ} \mathrm{C}$, a solution of phosphate $1(0.11 \mathrm{mmol})$ in THF $(1.1 \mathrm{~mL})$ was added. The reaction progress was monitored by TLC (products are baseline in EtOAc), and upon completion ( 2 hours) were quenched with $10 \% \mathrm{HCl}$ aq. $(1 \mathrm{~mL})$ and stirred for 30-60 min. The layers were separated and the aqueous layer extracted with $\mathrm{CH}_{2} \mathrm{Cl}_{2}(4 \times 1 \mathrm{~mL})$ and the combined organic layers were subsequently washed with $\mathrm{H}_{2} \mathrm{O}(1 \times 1 \mathrm{~mL})$ and the resulting white solids were filtered off. The aqueous layer was removed and the organic layer was concentrated under reduced pressure. The crude acids were used without further purification.

\section{General Procedure for Making Alkyl Zinc Iodides (Cy-Zn-I):}

To a heterogeneous mixture of activated $\mathrm{Zn}$ dust $(288 \mathrm{mg}, 4.4 \mathrm{mmol})$ in THF (330 $\mu \mathrm{L})$ under argon was added dibromoethane $(15 \mu \mathrm{L}, 0.176 \mathrm{mmol})$, which was heated to $65^{\circ} \mathrm{C}$ for one minute and then cooled to room temperature. Trimethylsilyl chloride was then added (16 $\mu \mathrm{L}, 0.132$ $\mathrm{mmol})$ and the mixture was allowed to stir for 15 minutes, upon which time the appropriate alkyl iodide $(4 \mathrm{mmol})$ in THF $(1.6 \mathrm{~mL})$ was added and the reaction heated to $40{ }^{\circ} \mathrm{C}$ with stirring. Upon completion (monitored by GC or Zn disappearance), the reagent was used immediately.

General Procedure for Red-Al ${ }^{\circledR}$ of Phosphate Acids: A stirring solution of the phosphate acid $(0.124 \mathrm{mmol})$ in toluene $(1.24 \mathrm{~mL})$ was cooled to $0{ }^{\circ} \mathrm{C}$ and Red-Al $(151 \mu \mathrm{L}, 0.495 \mathrm{mmol})$ was added dropwise. The reaction was allowed to proceed for 30 minutes and monitored by TLC. If necessary, an additional 1 equivalent of Red-Al was added to ensure completion. Reaction was quenched with $\mathrm{H}_{2} \mathrm{O}(1 \mathrm{~mL})$ followed by slow addition of $10 \% \mathrm{HCl}$ aq. $(1 \mathrm{~mL})$ at $0{ }^{\circ} \mathrm{C}$. The layers 
were separated and the aqueous layer extracted with $\mathrm{CH}_{2} \mathrm{Cl}_{2}(4 \mathrm{x})$ and the combined organic layers were subsequently washed with brine and dried $\left(\mathrm{MgSO}_{4}\right)$.

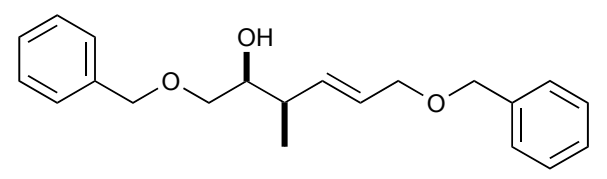

(2S,3R,E)-1,6-bis(benzyloxy)-3-methylhex-4-en-2-ol 9a: Following procedure A (100 mg of 1) and phosphate acid removal, column chromatography (Heptane:EtOAc 4:1) afforded 9a (67 $\mathrm{mg}, 83 \%)$ as a clear oil over two steps. $\quad[\alpha]_{\mathrm{D}}+30.4\left(c=1.0, \mathrm{CH}_{2} \mathrm{Cl}_{2}\right)$; IR (neat) 3461,2358 , 1456, 1361, 1249, $1027 \mathrm{~cm}^{-1}$; ${ }^{1} \mathrm{H}$ NMR (400 MHz, $\left.\mathrm{CDCl}_{3}\right) \delta$ 7.35-7.26 (m, 10H), 5.58-5.79 (m, $2 \mathrm{H}), 4.53(\mathrm{~d}, J=1.8 \mathrm{~Hz}, 2 \mathrm{H}), 4.48(\mathrm{~s}, 2 \mathrm{H}), 3.96-3.97(\mathrm{~m}, 2 \mathrm{H}), 3.64$ (ddd, $J=7.2,7.2,3.0 \mathrm{~Hz}$, $1 \mathrm{H}), 3.55$ (dd, $J=9.5,3.1,1 \mathrm{H}), 3.39$ (dd, $J=9.7,7.7,1 \mathrm{H}), 2.32-2.39$ (m, 1H), 1.09 (d, $J=6.8$ $\mathrm{Hz}, 3 \mathrm{H}) ;{ }^{13} \mathrm{C} \mathrm{NMR}\left(100 \mathrm{MHz}, \mathrm{CDCl}_{3}\right) \delta 138.26,137.90,135.62,128.44,127.77,127.71,127.59$, $127.05,73.53,73.34,72.66,71.98,70.60,39.80,15.9$. HRMS calculated for $\mathrm{C}_{21} \mathrm{H}_{26} \mathrm{O}_{3}(\mathrm{M}+\mathrm{Na})^{+}$ 349.1780; found 349.1817 (FAB).

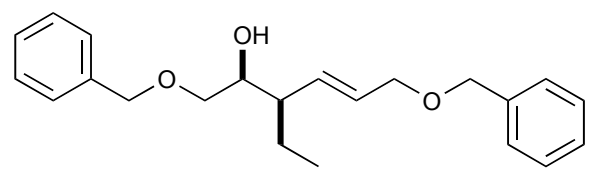

(2S,3R,E)-1,6-bis(benzyloxy)-3-ethylhex-4-en-2-ol 9b: Following procedure A (50 mg of 1) and phosphate acid removal, column chromatography (Heptane:EtOAc 4:1) afforded 9b (38 mg, $90 \%)$ as a clear oil over two steps; $[\alpha]_{\mathrm{D}}+4.87\left(c=0.58, \mathrm{CH}_{2} \mathrm{Cl}_{2}\right)$; IR (neat) $3420,2914,1457$, 1269, 1101, 1073, 737, $699 \mathrm{~cm}^{-1} ;{ }^{1} \mathrm{H}$ NMR (400 MHz, $\left.\mathrm{CDCl}_{3}\right) \delta$ 7.35-7.26 (m, 10H), $5.62(\mathrm{dt}, J$ $=15.4,6 \mathrm{~Hz}, 1 \mathrm{H}), 5.44(\mathrm{dd}, J=15.4,9.3 \mathrm{~Hz}, 1 \mathrm{H}), 4.53$ (d, $J=4.4 \mathrm{~Hz}, 2 \mathrm{H}), 4.49$ (s, 2H), 3.99 (d, $J=5.9 \mathrm{~Hz}, 2 \mathrm{H}), 3.7-3.66(\mathrm{~m}, 1 \mathrm{H}), 3.57(\mathrm{~d}, J=9.5 \mathrm{~Hz}, 1 \mathrm{H}), 3.38(\mathrm{t}, J=9.3 \mathrm{~Hz}, 1 \mathrm{H}), 2.48(\mathrm{~s}, 1 \mathrm{H})$, 2.14-2.06 (m, 1H), 1.32-1.21 (m, 2H), $0.88(\mathrm{t}, J=7.5 \mathrm{~Hz}, 3 \mathrm{H}) ;{ }^{13} \mathrm{C} \mathrm{NMR}\left(100 \mathrm{MHz}, \mathrm{CDCl}_{3}\right) \delta$ 138.26, 137.89, 133.60, 129.01, 128.51, 128.41, 128.36, 127.75, 127.72, 127.69, 127.57, 126.94, 73.32, 72.94, 72.49, 71.77, 70.50, 47.92, 23.32, 11.56. HRMS calculated for $\mathrm{C}_{22} \mathrm{H}_{28} \mathrm{O}_{3}$ $\left(\mathrm{M}+\mathrm{NH}_{4}\right)^{+}$358.2382; found $358.2381(\mathrm{FAB})$. 


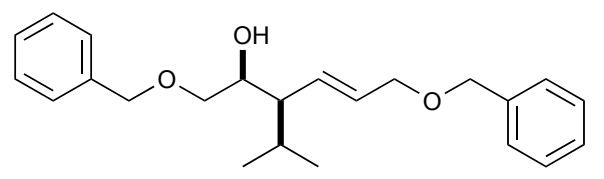

(2S,3R,E)-1,6-bis(benzyloxy)-3-isopropylhex-4-en-2-ol 9c: Following procedure A (50 mg of 1) and phosphate acid removal, column chromatography (Heptane:EtOAc 5:1) afforded 9c (44.5 mg, $89 \%$ ) as a clear oil over two steps. $[\alpha]_{D}+17.5,\left(c, 2.1 \mathrm{CH}_{2} \mathrm{Cl}_{2}\right)$; IR (neat) 3446, 2348, 1101 $\mathrm{cm}^{-1} ;{ }^{1} \mathrm{H}$ NMR $\left(400 \mathrm{MHz}, \mathrm{CDCl}_{3}\right) \delta$ 7.38-7.27 (m, 10H), 5.59 (ddd, $\left.J=15.6,6.0,6.0 \mathrm{~Hz}, 1 \mathrm{H}\right)$, $5.43(\mathrm{dd}, J=15.4,10.0 \mathrm{~Hz}, 1 \mathrm{H}), 4.53(\mathrm{dd}, J=23.0,11.7 \mathrm{~Hz}, 2 \mathrm{H}), 4.48$ (dd, $J=12.2,8.0 \mathrm{~Hz}, 2 \mathrm{H})$ $3.92(\mathrm{dd}, J=5.9,1.0 \mathrm{~Hz}, 2 \mathrm{H}), 3.78-3.84(\mathrm{~m}, 1 \mathrm{H}), 3.57(\mathrm{dd}, J=9.5,2.6 \mathrm{~Hz}, 1 \mathrm{H}), 3.33(\mathrm{dd}, J=$ 9.4, $7.6 \mathrm{~Hz}, 1 \mathrm{H}), 2.39$ (bs, 1H), 2.13-2.21 (m, 1H), 2.07 (ddd, $J=9.6,9.6,3.1 \mathrm{~Hz}, 1 \mathrm{H}), 0.88$ (d, $J$ $=6.8 \mathrm{~Hz}, 3 \mathrm{H}), 0.86(\mathrm{~d}, J=6.9 \mathrm{~Hz}, 3 \mathrm{H}) ;{ }^{13} \mathrm{C} \mathrm{NMR}\left(100 \mathrm{MHz}, \mathrm{CDCl}_{3}\right) \delta 138.28,137.90,130.41$, $130.11,128.42,128.37,127.75,127.72$, 127.70, 127.58, 73.49, 73.34, 71.70, 70.46, 70.04, 51.79, 26.66, 21.38, 16.82. HRMS calculated for $\mathrm{C}_{23} \mathrm{H}_{30} \mathrm{O}_{3}(\mathrm{M}+\mathrm{Na})^{+}$377.2093; found 377.2075 (FAB).

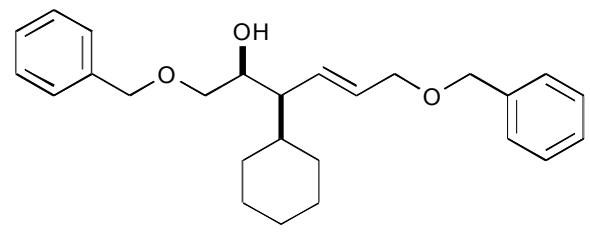

(2S,3R,E)-1,6-bis(benzyloxy)-3-cyclohexylhex-4-en-2-ol 9d: Following procedure B (100 mg of 1) and phosphate acid removal, column chromatography (Heptane:EtOAc 5:1) afforded 9d (68 mg, $70 \%$ ) as a clear oil; $[\alpha]_{\mathrm{D}}+9.84\left(c=1.58, \mathrm{CH}_{2} \mathrm{Cl}_{2}\right)$; IR (neat) 3453, 3031, 2922, 2850, 1496, 1450, 1098, 737, $698 \mathrm{~cm}^{-1} ;{ }^{1} \mathrm{H}$ NMR (500 MHz, $\left.\mathrm{CDCl}_{3}\right) \delta$ 7.37-7.28 (m, 10H), 5.57 (dt, $J$ $=15.4,5.9 \mathrm{~Hz}, 1 \mathrm{H}), 5.44(\mathrm{dd}, J=15.4,10 \mathrm{~Hz}, 1 \mathrm{H}), 4.53(\mathrm{dd}, J=11.8,10.6 \mathrm{~Hz}, 2 \mathrm{H}), 4.49$ (s, 2H), $3.98(\mathrm{~d}, J=5.9 \mathrm{~Hz}, 2 \mathrm{H}), 3.86(\mathrm{td}, J=8.4,2.5 \mathrm{~Hz}, 1 \mathrm{H}), 3.57(\mathrm{dd}, J=9.5,2.6 \mathrm{~Hz}, 1 \mathrm{H}), 3.32$ $(\mathrm{dd}, J=9.4,7.8 \mathrm{~Hz}, 1 \mathrm{H}), 2.42$ (d, $J=4 \mathrm{~Hz}, 1 \mathrm{H}), 2.07$ (td, $J=9.6,3.9 \mathrm{~Hz}, 1 \mathrm{H}), 1.80-1.70$ (m, 4H), 1.65 (d, $J=12.7 \mathrm{~Hz}, 1 \mathrm{H}), 1.50$ (d, $J=12.8 \mathrm{~Hz}, 1 \mathrm{H}), 1.33-1.20$ (m, 2H), 1.14-1.06 (m, 2H), $0.94(\mathrm{ddd}, J=13,12.8,3.1 \mathrm{~Hz}, 1 \mathrm{H}) ;{ }^{13} \mathrm{C} \mathrm{NMR}\left(125 \mathrm{MHz}, \mathrm{CDCl}_{3}\right) \delta 138.57,138.19,131.42$, $130.15,128.66,128.62,127.99,127.95,127.93,127.83,73.30,73.58,72.01,70.73,69.68,52.07$, 37.37, 32.16, 28.09, 26.90, 26.84, 26.75; HRMS calculated for $\mathrm{C}_{26} \mathrm{H}_{34} \mathrm{O}_{3}(\mathrm{M}+\mathrm{Li})^{+} 401.2668$; found $401.2678(\mathrm{FAB})$. 


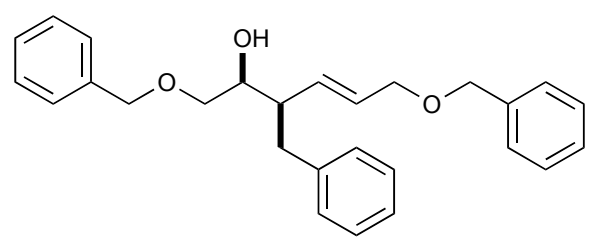

(2S,3R,E)-3-benzyl-1,6-bis(benzyloxy)hex-4-en-2-ol 9e: Following procedure B (100 mg 1) and phosphate acid removal, column chromatography (Heptane:EtOAc 4:1) afforded 9e (81.0 mg, 82\%) as a clear oil over two steps. $[\alpha]_{\mathrm{D}}-6.2\left(c 1.4, \mathrm{CH}_{2} \mathrm{Cl}_{2}\right)$; IR (neat) 3444, 1494, 1452, $1097 \mathrm{~cm}^{-1} ;{ }^{1} \mathrm{H}$ NMR (400 MHz, $\left.\mathrm{CDCl}_{3}\right) \delta$ 7.38-7.15 (m, 15H), $5.48(\mathrm{dd}, J=15.7,8.1 \mathrm{~Hz}, 1 \mathrm{H})$, $5.43(\mathrm{ddd}, J=15.7,10.6,5.3 \mathrm{~Hz}, 1 \mathrm{H}), 4.56(\mathrm{dd}, J=11.9,7.3 \mathrm{~Hz} 2 \mathrm{H}), 4.31(\mathrm{dd}, J=12.0,0.1 \mathrm{~Hz}$, 2H), $3.91(\mathrm{dd}, J=12.8,4.1 \mathrm{~Hz}, 1 \mathrm{H}), 3.87$ (dd, $J=12.3,5.0 \mathrm{~Hz}, 1 \mathrm{H}), 3.78-3.73(\mathrm{~m}, 1 \mathrm{H}), 3.65-$ 3.57 (m, 1H), 3.50-3.43 (m, 1H), 3.12 (dd, $J=13.4,4.0 \mathrm{~Hz}, 1 \mathrm{H}), 2.68$ (dd, $J=13.5,9.3 \mathrm{~Hz}, 1 \mathrm{H})$, 2.62-2.52 (m, 2H); ${ }^{13} \mathrm{C}$ NMR (100 MHz, $\left.\mathrm{CDCl}_{3}\right) \delta 139.79,138.31,137.00,132.81,129.52$, $129.38,128.45,128.31,128.07,127.82,127.76,127.67,127.51,125.85,73.37,72.68,71.81$, 71.37, 70.27, 47.82, 37.27. HRMS calculated for $\mathrm{C}_{37} \mathrm{H}_{30} \mathrm{O}_{3}(\mathrm{M}+\mathrm{Na})^{+}$425.2093; found 425.2083 (FAB).

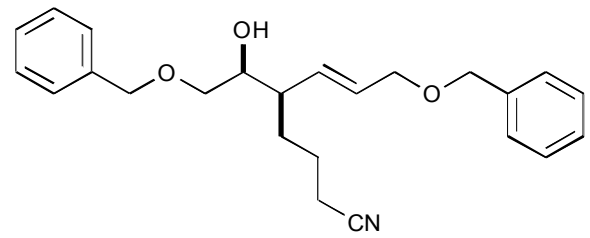

\section{(R,E)-8-(benzyloxy)-5-((S)-2-(benzyloxy)-1-hydroxyethyl)oct-6-enenitrile 9f:}

Following procedure B (100 $\mathrm{mg}$ of 1) and methylation/phosphate removal, column chromatography (Heptane:EtOAc 4:1) afforded 9f $(78.0 \mathrm{mg}, 83 \%)$ as a clear oil. $[\alpha]_{\mathrm{D}}=-12.0(c$ $=1.5, \mathrm{CH}_{2} \mathrm{Cl}_{2}$ ); IR (neat) 3463, 3031, 2927, 2246, 742, $702 \mathrm{~cm}^{-1} ;{ }^{1} \mathrm{H}$ NMR $\left(400 \mathrm{MHz}, \mathrm{CDCl}_{3}\right) \delta$ 7.36-7.28 (m, 10H), $5.63(\mathrm{ddd}, J=15.5,5.7,5.7 \mathrm{~Hz}, 1 \mathrm{H}), 5.43(\mathrm{dd}, J=15.5,9.5 \mathrm{~Hz}, 1 \mathrm{H}), 4.52$ $(\mathrm{dd}, J=11.8,7.7 \mathrm{~Hz}, 2 \mathrm{H}), 4.48(\mathrm{dd}, J=9.1,0.1 \mathrm{~Hz}, 2 \mathrm{H}), 3.97(\mathrm{~d}, J=5.6 \mathrm{~Hz}, 2 \mathrm{H}), 3.62$ (ddd, $J=$ 7.4, 5.4, 2.3 Hz, 1H), 3.55 (dd, $J=9.5,9.4 \mathrm{~Hz}, 1 \mathrm{H}), 3.36$ (dd, $J=9.4,7.6 \mathrm{~Hz}, 1 \mathrm{H}), 2.47$ (bs, 1H), 2.32 (dddd, $J=14.3,7.3,7.0,2.6 \mathrm{~Hz} 2 \mathrm{H}), 2.18$ (ddd, $J=18.2,10.1,3.3 \mathrm{~Hz}, 1 \mathrm{H}), 1.96-1.86$ (m, $1 \mathrm{H}), 1.78-1.67(\mathrm{~m}, 1 \mathrm{H}), 1.55(\mathrm{ddd}, J=6.9,6.0,4.8 \mathrm{~Hz}, 1 \mathrm{H}), 1.34-1.45(\mathrm{~m}, 1 \mathrm{H}) ;{ }^{13} \mathrm{C}$ NMR $(100$ $\left.\mathrm{MHz}, \mathrm{CDCl}_{3}\right) \delta 138.17,137.78,132.42,129.90,128.51,128.46,127.91,127.80,127.75,127.72$, 119.72, 73.44, 72.71, 72.49, 72.23, 70.30, 45.91, 29.76, 23.31, 17.27. HRMS calculated for $\mathrm{C}_{24} \mathrm{H}_{29} \mathrm{NO}_{3}\left(\mathrm{M}+\mathrm{NH}_{4}\right)^{+}$397.2491; found 397.2483 (FAB). 


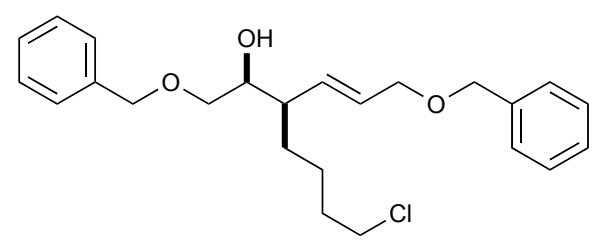

\section{(2S,3R)-1-(benzyloxy)-3-((E)-3-(benzyloxy)prop-1-enyl)-7-chloroheptan-2-ol 9g:}

Following procedure B (100mg of 1) and methylation/phosphate removal, column chromatography (Heptane:EtOAc 5:1) afforded $9 \mathrm{~g}(84.7 \mathrm{mg}, 85 \%)$ as a clear oil. $[\alpha]_{\mathrm{D}}=+5.8(c$ $=0.625, \mathrm{CH}_{2} \mathrm{Cl}_{2}$ ); IR (neat) 3442, 3031, 2921, 2856, 1494, 1452, 1361, 1099, 736, $698 \mathrm{~cm}^{-1} ;{ }^{1} \mathrm{H}$ NMR (400 MHz, $\left.\mathrm{CDCl}_{3}\right) \delta$ 7.32-7.11 (m, 10H), 5.67-5.59 (m, 1H), $5.46(\mathrm{dd}, J=15.5,9.4 \mathrm{~Hz}$, 1H), 4.54 (dd, $J=17.4,11.9 \mathrm{~Hz}, 2 \mathrm{H}), 4.49$ (app s, 2H), 3.99 (dd, $J=5.9,1.1 \mathrm{~Hz}, 2 \mathrm{H}), 3.66$ (ddd, $J=7.8,7.8,2.9 \mathrm{~Hz}, 1 \mathrm{H}), 3.57(\mathrm{dd}, J=9.5,2.9 \mathrm{~Hz}, 1 \mathrm{H}), 3.57-3.52(\mathrm{~m}, 2 \mathrm{H}), 3.39$ (dd, $J=9.5$, $7.5 \mathrm{~Hz}, 1 \mathrm{H}), 2.22-1.88(\mathrm{~m}, 3 \mathrm{H}), 1.84-1.69(\mathrm{~m}, 3 \mathrm{H}), 1.48-1.24(\mathrm{~m}, 4 \mathrm{H}) ;{ }^{13} \mathrm{C} \mathrm{NMR}(100 \mathrm{MHz}$, $\left.\mathrm{CDCl}_{3}\right) \delta 138.27,137.86,133.35,129.24,128.45,128.38,127.73,127.70,127.61,73.38,72.71$, $72.85,72.62,71.90,70.3420,46.24,45.00,35.59,29.70,24.43$. HRMS calculated for $\mathrm{C}_{24} \mathrm{H}_{31} \mathrm{ClO}_{3}\left(\mathrm{M}+\mathrm{NH}_{4}\right)^{+} 420.2305$; found 420.2310 (FAB).

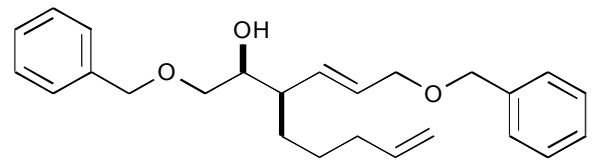

(2S,3R,E)-1-(benzyloxy)-3-(3-(benzyloxy)prop-1-enyl)oct-7-en-2-ol 9h: Following procedure B and phosphate acid removal, column chromatography (Heptane:EtOAc 4:1) afforded 9h (61 $\mathrm{mg}, 65 \%)$ as a clear oil; $[\alpha]_{\mathrm{D}}+3.53\left(c=0.17, \mathrm{CH}_{2} \mathrm{Cl}_{2}\right)$; IR (neat) $3422,1274,750,699 \mathrm{~cm}^{-1} ;{ }^{1} \mathrm{H}$ $\operatorname{NMR}\left(500 \mathrm{MHz}, \mathrm{CDCl}_{3}\right) \delta$ 7.36-7.30 (m, 10H), $5.80(\mathrm{ddt}, J=17,10.2,6.6 \mathrm{~Hz}, 1 \mathrm{H}), 5.62(\mathrm{dt}, J=$ 15.5, $6 \mathrm{~Hz}, 1 \mathrm{H}), 5.46(\mathrm{dd}, J=15.5,9.4 \mathrm{~Hz}, 1 \mathrm{H}), 5.00(\mathrm{dd}, J=17.1,1.4 \mathrm{~Hz}, 1 \mathrm{H}), 4.94(\mathrm{dd}, J=$ 10.2, $1 \mathrm{~Hz}, 1 \mathrm{H}), 4.54(\mathrm{dd}, 18.3,11.7,2 \mathrm{H}), 4.49$ (s, 2H), $3.99(\mathrm{~d}, J=6 \mathrm{~Hz}, 2 \mathrm{H}), 3.66(\mathrm{td}, J=7.7$, $3 \mathrm{~Hz}, 1 \mathrm{H}), 3.57(\mathrm{dd}, J=9.5,3 \mathrm{~Hz}, 1 \mathrm{H}), 3.38\left(\mathrm{dd}, J_{\mathrm{HH}}=9.3,7.7 \mathrm{~Hz}, 1 \mathrm{H}\right), 2.40$ (d, $J=4.2 \mathrm{~Hz}$, 1H), $2.19(\mathrm{ddd}, J=9.4,7.9,3.6 \mathrm{~Hz}, 1 \mathrm{H}), 2.09-2.00(\mathrm{~m}, 2 \mathrm{H}), 1.77-1.73(\mathrm{~m}, 1 \mathrm{H}), 1.49-1.45$ (m, 1H), 1.31-1.27 (m, 2H); ${ }^{13} \mathrm{C}$ NMR (125 MHz, $\left.\mathrm{CDCl}_{3}\right) \delta$ 139.07, 138.53, 138.15, 133.98, 129.20, $128.67,128.61,128.01,127.95,127.94,127.82,114.65,73.59,73.10,72.94,72.06,70.72,46.50$, 34.03, 30.19, 26.73. HRMS calculated for $\mathrm{C}_{25} \mathrm{H}_{32} \mathrm{O}_{3}\left(\mathrm{M}+\mathrm{NH}_{4}\right)^{+}$398.2695; found 398.2696 (FAB). 


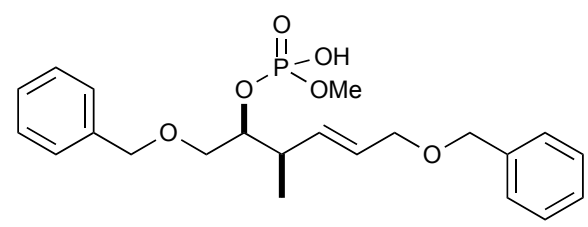

(2S,3R,E)-1,6-bis(benzyloxy)-3-methylhex-4-en-2-yl methyl hydrogen phosphate 8a: Using procedure A (50 $\mathrm{mg}$ of $\mathbf{1}$ ) afforded $\mathbf{8 a}(51.5 \mathrm{mg}, 99 \%)$ as a clear viscous oil (compound was processed without further purification). $[\alpha]_{\mathrm{D}}+15.6\left(c=4.5, \mathrm{CH}_{2} \mathrm{Cl}_{2}\right)$; IR (neat) 2331, 1496, 1454, $1232 \mathrm{~cm}^{-1}$; ${ }^{1} \mathrm{H}$ NMR (400 MHz, $\left.\mathrm{CDCl}_{3}\right) \delta 10.16$ (bs, $\left.1 \mathrm{H}\right), 7.37-7.26(\mathrm{~m}, 10 \mathrm{H}), 5.71-5.62$ (m, 2H), 4.58-4.49 (m, 2H), $4.48(\mathrm{~s}, 2 \mathrm{H}), 4.36-4.32(\mathrm{~m}, 1 \mathrm{H}), 3.96(\mathrm{~s}, 2 \mathrm{H}), 3.71-3.53(\mathrm{~m}, 5 \mathrm{H})$, 2.73-2.61 (m, 1H), $1.11(\mathrm{~d}, J=6.7 \mathrm{~Hz}, 3 \mathrm{H}) ;{ }^{13} \mathrm{C} \mathrm{NMR}\left(100 \mathrm{MHz}, \mathrm{CDCl}_{3}\right) \delta$ 138.67, 138.30, $134.72,128.81,128.80,128.25,128.22,128.11,128.05,81.45,73.71,72.44,71.02,70.45$, 54.61, 38.80, 15.87; ${ }^{31} \mathrm{P}$ NMR (162 MHz, $\left.\mathrm{CDCl}_{3}\right) \delta$ 2.67. HRMS calculated for $\mathrm{C}_{22} \mathrm{H}_{29} \mathrm{O}_{6} \mathrm{P}$ $(\mathrm{M}+\mathrm{Na})^{+}$443.1599; found $443.1600(\mathrm{FAB})$.

\section{General Methylation procedure:}

To a stirring solution of phosphate acid $(0.295 \mathrm{mmol})$ in $\mathrm{MeOH}(0.6 \mathrm{~mL})$ was slowly added (trimethylsilyl)diazomethane $(2.0 \mathrm{M}, 1.48 \mathrm{mmol})$. Upon addition, the reaction was allowed to run for 20 minutes at room temperature with stirring to ensure completion (monitored by TLC), then quenched by dropwise addition of acetic acid until effervescence ceases. The reaction was concentrated under reduced pressure and pushed through a small plug of silica (4:1 Hexanes/EtOAc) to supply the desired methyl phosphate ester.

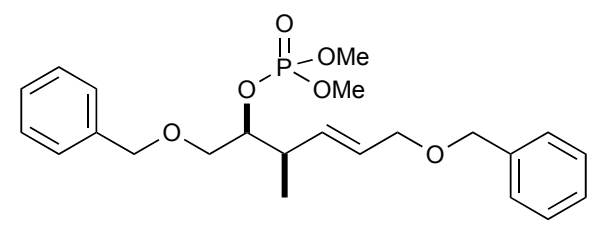

(2S,3R,E)-1,6-bis(benzyloxy)-3-methylhex-4-en-2-yl dimethyl phosphate 10a: Using procedure A, methylation followed by column chromatography (EtOAc:Heptane 2:1) afforded 10a $(50 \mathrm{mg}, 95 \%)$ as a clear oil over two steps. $[\alpha]_{\mathrm{D}}-27.0\left(c=2.0, \mathrm{CH}_{2} \mathrm{Cl}_{2}\right)$; IR (neat) 3062, 2952, 1454, 1363, 1278, $1041 \mathrm{~cm}^{-1}$; ${ }^{1} \mathrm{H}$ NMR $\left(400 \mathrm{MHz}, \mathrm{CDCl}_{3}\right) \delta$ 7.36-7.24 (m, 10H), 5.69$5.62(\mathrm{~m}, 2 \mathrm{H}), 4.58-4.40(\mathrm{~m}, 5 \mathrm{H}), 3.97-3.94(\mathrm{~m}, 2 \mathrm{H}), 3.73\left(\mathrm{~d}, J_{\mathrm{HP}}=11.2,2 \mathrm{H}\right), 3.69\left(\mathrm{~d}, J_{\mathrm{HP}}=11.2\right.$, 2H), 3.64-3.56 (m, 2H), 2.73-2.64 (m, $1 \mathrm{H}), 1.10(\mathrm{~d}, J=6.9 \mathrm{~Hz}, 3 \mathrm{H}) ;{ }^{13} \mathrm{C}$ NMR $(125 \mathrm{MHz}$, $\left.\mathrm{CDCl}_{3}\right) \delta 138.19,137.79,134.00,128.34,128.31,127.89,127.71,127.69,127.65,127.57,80.95$ 
$\left(\mathrm{d}, J_{\mathrm{CP}}=6.3\right), 73.16,71.99,70.55,70.07\left(\mathrm{~d}, J_{\mathrm{CP}}=1.3\right), 54.28\left(\mathrm{~d}, J_{\mathrm{CP}}=6.3\right), 54.23\left(\mathrm{~d}, J_{\mathrm{CP}}=6.3\right)$, $38.44\left(\mathrm{~d}, J_{\mathrm{CP}}=5.0\right), 15.43 ;{ }^{31} \mathrm{P}$ NMR $\left(162 \mathrm{MHz}, \mathrm{CDCl}_{3}\right) \delta 2.06$; HRMS calculated for $\mathrm{C}_{23} \mathrm{H}_{32} \mathrm{O}_{6} \mathrm{P}(\mathrm{M}+\mathrm{H})^{+} 435.1937$; found 435.1967 (FAB).

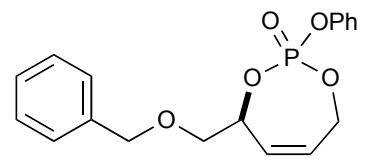

(S)-configured unsymmetrical monocyclic phosphate 11: A flask containing allylic alcohol 3 $(0.680 \mathrm{~g}, 3.83 \mathrm{mmol})$ was diluted up in THF $(0.2 \mathrm{M}, 20 \mathrm{~mL})$ and cooled to $-40{ }^{\circ} \mathrm{C}$, followed by dropwise addition of BuLi (1.53, 2.5M). After 10 minutes of stirring, allyldiphenyl phosphate $(1.45 \mathrm{~g}, 5 \mathrm{mmol})$ in THF (1M, $5 \mathrm{~mL})$ was cannulated to the flask containing the alkoxide. The solution was stirred for eight hours while slowly warming to room temperature. The reaction was quenched with sat'd aq. $\mathrm{NH}_{4} \mathrm{Cl}^{-}$and the layers were separated and the aqueous layer extracted with $\mathrm{Et}_{2} \mathrm{O}(2 \mathrm{x})$. The combined organic layers were concentrated under reduced pressure and dried $\left(\mathrm{Na}_{2} \mathrm{SO}_{4}\right)$. Column chromatography (1:1 Hex:EtOAc) afforded the acyclic phosphate ester $(1.03 \mathrm{~g}, 71 \%)$ as an oil. To a flask containing the phosphate precursor was added degassed $\mathrm{CH}_{2} \mathrm{Cl}_{2}(0.05 \mathrm{M}, 22.6 \mathrm{~mL})$ and was subsequently fitted with a reflux condenser and needle, through which a slow steady stream of $\mathrm{Ar}$ flowed. Grubbs catalyst $\left(\mathrm{ImesH}_{2}\right)\left(\mathrm{PCy}_{3}\right)\left(\mathrm{Cl}_{2}\right) \mathrm{Ru}=\mathrm{CHPh}(0.034 \mathrm{mg}, 0.04 \mathrm{mmol})$ was added and the reaction lowered into an oil bath warmed to $50{ }^{\circ} \mathrm{C}$. The reaction was monitored by TLC and after 1.0-1.5 hrs was deemed complete. Following cooling to room temperature, the reaction was concentrated and purified via column chromatograpy (2:1 Hex:EtOAc), to yield cyclic phosphate $\mathbf{1 1}$ as a 1:1 mixture of diastereomers at phosphorus $(0.301 \mathrm{~g}, 76 \%) ;{ }^{1} \mathrm{H}$ NMR $\left(400 \mathrm{MHz}, \mathrm{CDCl}_{3}\right) \delta$ 7.40-7.17 (m, 10H), 5.89-5.74 (m, 2H), 5.41-5.23 (m, 1H), 5.14-4.89 (m, 1H), 4.71-4.51 (m, 3H), 3.79-3.62 $(\mathrm{m}, 2 \mathrm{H}) ;{ }^{13} \mathrm{C}$ NMR $\left(100 \mathrm{MHz}, \mathrm{CDCl}_{3}\right) \delta 150.38\left(\mathrm{~d}, J_{\mathrm{CP}}=16.1\right), 150.22\left(\mathrm{~d}, J_{\mathrm{CP}}=16.1\right), 137.44$, $129.68\left(\mathrm{~d}, J_{\mathrm{CP}}=4.0\right), 128.61,128.38,128.23,127.78,127.67\left(\mathrm{~d}, J_{\mathrm{CP}}=7.0\right), 127.42,127.08$, $125.22,\left(\mathrm{~d}, J_{\mathrm{CP}}=6.0\right), 120.11\left(\mathrm{~d}, J_{\mathrm{CP}}=5.0\right), 119.87\left(\mathrm{~d}, J_{\mathrm{CP}}=4.0\right), 74.35\left(\mathrm{~d}, J_{\mathrm{CP}}=7.0\right), 74.01(\mathrm{~d}$, $\left.J_{\mathrm{CP}}=4.0\right), 73.47,73.28,71.58\left(\mathrm{~d}, J_{\mathrm{CP}}=9.1\right), 71.15\left(\mathrm{~d}, J_{\mathrm{CP}}=11.1\right), 64.87\left(\mathrm{~d}, J_{\mathrm{CP}}=7.0\right), 64.80(\mathrm{~d}$, $\left.J_{\mathrm{CP}}=8.0\right) ;{ }^{31} \mathrm{P}$ NMR $\left(162 \mathrm{MHz}, \mathrm{CDCl}_{3}\right) \delta-3.61$ and -2.87 ; HRMS calculated for $\mathrm{C}_{18} \mathrm{H}_{19} \mathrm{O}_{5} \mathrm{P}$ $(\mathrm{M}+\mathrm{Na})^{+}$369.0868; found $369.0872(\mathrm{FAB})$. 


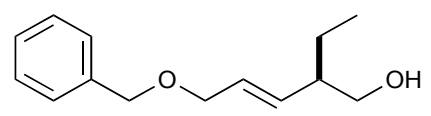

$(\boldsymbol{R}, \boldsymbol{E})$-5-(benzyloxy)-2-ethylpent-3-en-1-ol 12: Following procedure A (90 $\mathrm{mg}$ of 11) and methylation/phosphate removal, column chromatography (Heptane:EtOAc 3:1) afforded 12 (42.1 mg, 81\%) as a clear oil. $[\alpha]_{\mathrm{D}}+6.2\left(c=1.6, \mathrm{CH}_{2} \mathrm{Cl}_{2}\right)$; IR (neat) 3389, 2958, 1454, 1307, 1089, $1049 \mathrm{~cm}^{-1} ;{ }^{1} \mathrm{H}$ NMR $\left(400 \mathrm{MHz}, \mathrm{CDCl}_{3}\right) \delta$ 7.22-7.19 (m, 5H), $5.63(\mathrm{dt}, J=15.4,6.0 \mathrm{~Hz}, 1 \mathrm{H}), 5.43$ $(\mathrm{dd}, J=15.5,8.7 \mathrm{~Hz}, 1 \mathrm{H}), 4.45(\mathrm{~s}, 2 \mathrm{H}), 3.49(\mathrm{dd}, J=10.6,5.2 \mathrm{~Hz}, 1 \mathrm{H}), 3.36(\mathrm{dd}, J=10.6,8.0$ $\mathrm{Hz}, 1 \mathrm{H}) 2.12-2.03(\mathrm{~m}, 1 \mathrm{H}), 1.71(\mathrm{bs}, 1 \mathrm{H}), 1.45-1.34(\mathrm{~m}, 1 \mathrm{H}), 1.26-1.15(\mathrm{~m}, 1 \mathrm{H}), 0.83(\mathrm{t}, J=7.5$ $\mathrm{Hz}, 3 \mathrm{H}) ;{ }^{13} \mathrm{C} \mathrm{NMR}\left(100 \mathrm{MHz}, \mathrm{CDCl}_{3}\right) \delta 138.24,135.15,129.18,128.43,127.68,127.58,72.11$, 70.67, 65.60, 47.26, 23.78, 11.67. HRMS calculated for $\mathrm{C}_{14} \mathrm{H}_{20} \mathrm{O}_{2}(\mathrm{M}+\mathrm{Na})^{+}$243.1361; found 243.1361 (FAB).

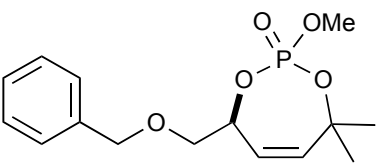

(S)-configured unsymmetrical monocyclic phosphate 14: A flask containing allylic alcohol 3 $(0.239 \mathrm{~g}, 1.34 \mathrm{mmol})$ was diluted up in THF $(0.2 \mathrm{M}, 6.7 \mathrm{~mL})$ and cooled to $-30{ }^{\circ} \mathrm{C}$, followed by dropwise addition of BuLi $(0.537 \mathrm{~mL}, 2.5 \mathrm{M})$. After 10 minutes of stirring, dichloromethyl phosphate $(0.147,1.47 \mathrm{mmol})$ was added to the flask containing the alkoxide. The solution was stirred for one hour while slowly warming to room temperature. The reaction was quenched with sat'd aq. $\mathrm{NH}_{4} \mathrm{Cl}^{-}$and the layers were separated and the aqueous layer extracted with $\mathrm{Et}_{2} \mathrm{O}$ (2x). The combined organic layers were concentrated under reduced pressure and dried $\left(\mathrm{Na}_{2} \mathrm{SO}_{4}\right)$. Column chromatography (4:1 Hex:EtOAc) afforded the monochloride phosphate ester $(0.311 \mathrm{~g}, 80 \%)$ as an oil. The chloride was immediately used. A flask containing alcohol $13(0.074 \mathrm{mg}, 0.861 \mathrm{mmol})$ was charged with THF $(0.2 \mathrm{M}, 4.31 \mathrm{~mL})$ and cooled to $-30{ }^{\circ} \mathrm{C}$, followed by dropwise addition of BuLi $(0.346 \mathrm{~mL}, 2.4 \mathrm{M})$. After 10 minutes of stirring, a solution of the previously generated monochloride phosphate ester $(0.200 \mathrm{~g}, 0.688 \mathrm{mmol})$ in THF $(0.7 \mathrm{M}, 1 \mathrm{~mL})$ was cannulated to the flask containing the alkoxide. The solution was stirred for $1.5 \mathrm{hrs}$ while slowly warming to room temperature. The reaction was quenched with sat'd aq. $\mathrm{NH}_{4}{ }^{+} \mathrm{Cl}^{-}$and the layers were separated and the aqueous layer extracted with $\mathrm{Et}_{2} \mathrm{O}(2 \mathrm{x})$. The combined organic layers were concentrated under reduced pressure and dried $\left(\mathrm{Na}_{2} \mathrm{SO}_{4}\right)$. Due to 
the instability of the acyclic phosphate, the reaction mixture was carried on without chromatography. To a flask containing the crude phosphate ester (assumed to be $0.688 \mathrm{mmol}$ ) was added degassed toluene $(0.02 \mathrm{M}, 34 \mathrm{~mL})$ and was subsequently fitted with a reflux condenser, and needle through which a slow steady stream of Ar flowed. Grubbs catalyst $\left(\mathrm{ImesH}_{2}\right)\left(\mathrm{PCy}_{3}\right)\left(\mathrm{Cl}_{2}\right) \mathrm{Ru}=\mathrm{CHPh}(0.029 \mathrm{mg}, 0.034 \mathrm{mmol})$ was added and the reaction lowered into an oil bath warmed to $90{ }^{\circ} \mathrm{C}$. The reaction was monitored by TLC and after $1.0 \mathrm{hr}$ was deemed complete. Following cooling to room temperature, the reaction was concentrated and purified via column chromatograpy (2:1 Hex:EtOAc), to yield cyclic phosphate $\mathbf{1 4}$ as a 1:1 mixture of diastereomers at phosphorus $(0.100 \mathrm{~g}, 47 \%)$. One diastereomer could be purified, and was used for characterization; IR (neat) 3029, 2966, 1101, 737, $698 \mathrm{~cm}^{-1} ;{ }^{1} \mathrm{H}$ NMR $\left(500 \mathrm{MHz}, \mathrm{CDCl}_{3}\right) \delta$ 7.35-7.27 (m, $5 \mathrm{H}), 5.63\left(\mathrm{dd}, J=12.1 \mathrm{~Hz}, J_{\mathrm{HP}}=2.3 \mathrm{~Hz}, 1 \mathrm{H}\right), 5.53\left(\mathrm{dd}, J=12.1 \mathrm{~Hz}, J_{\mathrm{HP}}=3.1 \mathrm{~Hz}\right.$, $1 \mathrm{H}), 4.61(\mathrm{dd}, J=12,4.1 \mathrm{~Hz}, 2 \mathrm{H}), 3.81\left(\mathrm{~d}, J_{\mathrm{HP}}=11.2 \mathrm{~Hz}, 3 \mathrm{H}\right), 3.67(\mathrm{dd}, J=10.2,6.9 \mathrm{~Hz}, 1 \mathrm{H})$, 3.57 (ddd, $J=10.2,5.2,1.9 \mathrm{~Hz}, 1 \mathrm{H}), 1.75(\mathrm{~s}, 3 \mathrm{H}), 1.48\left(\mathrm{~d}, J_{\mathrm{HP}}=2.5 \mathrm{~Hz}, 3 \mathrm{H}\right) ;{ }^{13} \mathrm{C}$ NMR $(125$ $\left.\mathrm{MHz}, \mathrm{CDCl}_{3}\right) \delta 137.90,137.66,128.46,127.68,124.16,82.10\left(\mathrm{~d}, J_{\mathrm{CP}}=5.0 \mathrm{~Hz}\right), 73.55,72.79(\mathrm{~d}$, $\left.J_{\mathrm{CP}}=6.3 \mathrm{~Hz}\right), 71.86\left(\mathrm{~d}, J_{\mathrm{CP}}=10.1 \mathrm{~Hz}\right), 54.13\left(\mathrm{~d}, J_{\mathrm{CP}}=5.0 \mathrm{~Hz}\right), 30.53\left(\mathrm{~d}, J_{\mathrm{CP}}=11.3 \mathrm{~Hz}\right), 29.32$; ${ }^{31} \mathrm{P}$ NMR $\left(162 \mathrm{MHz}, \mathrm{CDCl}_{3}\right) \delta 0.07$ and -0.90 .

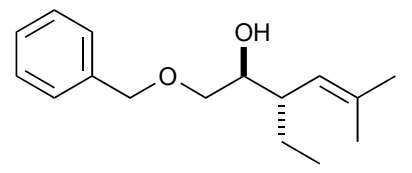

(2S,3S)-1-(benzyloxy)-3-ethyl-5-methylhex-4-en-2-ol 15: Following procedure A (90 mg of 14) and methylation/phosphate removal, column chromatography (Heptane:EtOAc 3:1) afforded $15(58.0 \mathrm{mg}, 81 \%)$ as a clear oil. $[\alpha]_{\mathrm{D}}=+13.1,\left(c=1.5, \mathrm{CH}_{2} \mathrm{Cl}_{2}\right)$; IR (neat) $3431,3029,2966$, $1101 \mathrm{~cm}^{-1} ;{ }^{1} \mathrm{H}$ NMR $\left(400 \mathrm{MHz}, \mathrm{CDCl}_{3}\right) \delta$ 7.31-7.22 (m, 5H), $5.02(\mathrm{~d}, J=8.9 \mathrm{~Hz}, 1 \mathrm{H}), 4.50(\mathrm{~s}$, 2H), 3.81-3.74 (m, 1H), 3.54 (dd, $J=9.6,3.1 \mathrm{~Hz}, 1 \mathrm{H}), 3.45$ (dd, $J=9.6,7.8,1 \mathrm{H}), 2.37-2.28(\mathrm{~m}$, 1H), 2.24 (bs, $1 \mathrm{H}), 1.78(\mathrm{~s}, 3 \mathrm{H}), 1.65,(\mathrm{~s}, 3 \mathrm{H}), 1.61-1.51(\mathrm{~m}, 1 \mathrm{H}), 1.34-1.24(\mathrm{~m}, 1 \mathrm{H}), 0.86$, (t, $J=$ 7.3, 3H); ${ }^{13} \mathrm{C}$ NMR $\left(100 \mathrm{MHz}, \mathrm{CDCl}_{3}\right) \delta 138.15,134.65,129.61,128.44,127.76,124.40$, $115.34,73.40,73.21,73.02,42.61,26.05,24.70,18.45$. 

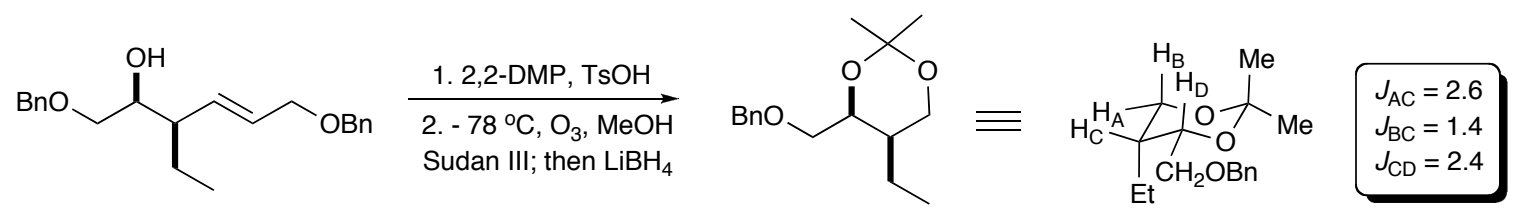

4-(benzyloxymethyl)-5-ethyl-2,2-dimethyl-1,3-dioxane: ${ }^{1} \mathrm{H}$ NMR $\left(400 \mathrm{MHz}, \mathrm{CDCl}_{3}\right) \delta 7.37$ $7.28(\mathrm{~m}, 5 \mathrm{H}), 4.62(\mathrm{~d}, J=12.1 \mathrm{~Hz}, 1 \mathrm{H}), 4.48(\mathrm{~d}, J=12.1 \mathrm{~Hz}, 1 \mathrm{H}), 4.26$ (ddd, $J=6.2,6.2,2.4$ $\mathrm{Hz}, 1 \mathrm{H}), 3.97$ (ddd, $J=11.9,2.6,1.11 \mathrm{~Hz}, 1 \mathrm{H}), 3.82$ (dd, $J=11.9,1.4 \mathrm{~Hz}, 1 \mathrm{H}), 3.49$ (dd, $J=$ 9.6, $6.6 \mathrm{~Hz}, 1 \mathrm{H}), 3.43$ (dd, $J=9.7,5.9 \mathrm{~Hz}, 1 \mathrm{H}), 1.77-1.65(\mathrm{~m}, 1 \mathrm{H}), 1.46(\mathrm{~s}, 3 \mathrm{H}), 1.38(\mathrm{~s}, 3 \mathrm{H})$, $1.35-1.27(\mathrm{~m}, 2 \mathrm{H}), 0.92(\mathrm{t}, J=7.2 \mathrm{~Hz})$.
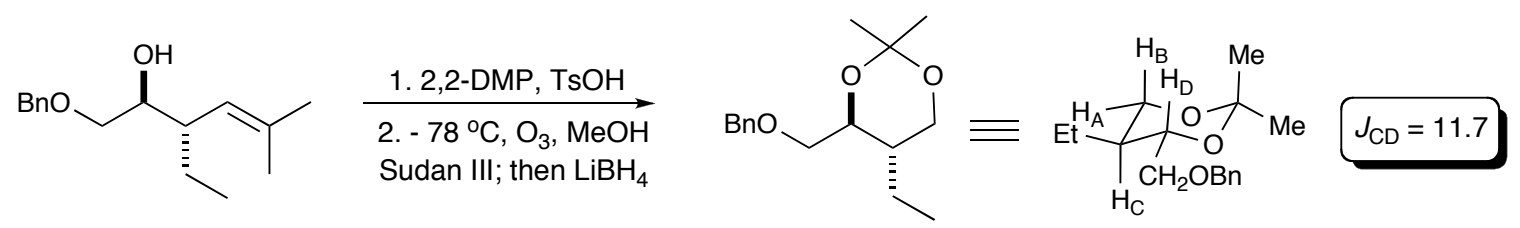

(4S,5S)-4-(benzyloxymethyl)-5-ethyl-2,2-dimethyl-1,3-dioxane: ${ }^{1} \mathrm{H}$ NMR (400 $\mathrm{MHz}, \mathrm{CDCl}_{3}$ )

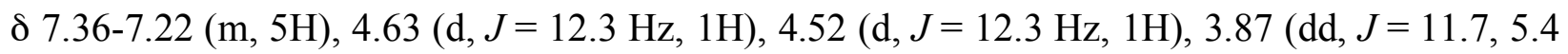
$\mathrm{Hz}, 1 \mathrm{H}), 3.74$ (ddd, $J=10.3,5.0,2.8 \mathrm{~Hz}, 1 \mathrm{H}), 85(\mathrm{t}, J=7.6 \mathrm{~Hz}, 3 \mathrm{H}) ;{ }^{13} \mathrm{C} \mathrm{NMR}(100 \mathrm{MHz}$, $\left.\mathrm{CDCl}_{3}\right) \delta$ 138.42, 128.33, 127.73, 127.56, 98.19, 73.58, 73.38, 71.50, 63.65, 37.02, 29.38, 21.08, 19.38, 10.05. HRMS calculated for $\mathrm{C}_{16} \mathrm{H}_{24} \mathrm{O}_{3}(\mathrm{M}+\mathrm{Na})^{+}$287.1623; found 287.1628 (FAB). 
NMR Spectra for Compounds 1,4-6, 8a, 9a-9h, 10a, 11,12,14,15:

$\operatorname{Bis}((S)$-1-(benzyloxy)but-3-en-2-yl) methyl phosphate

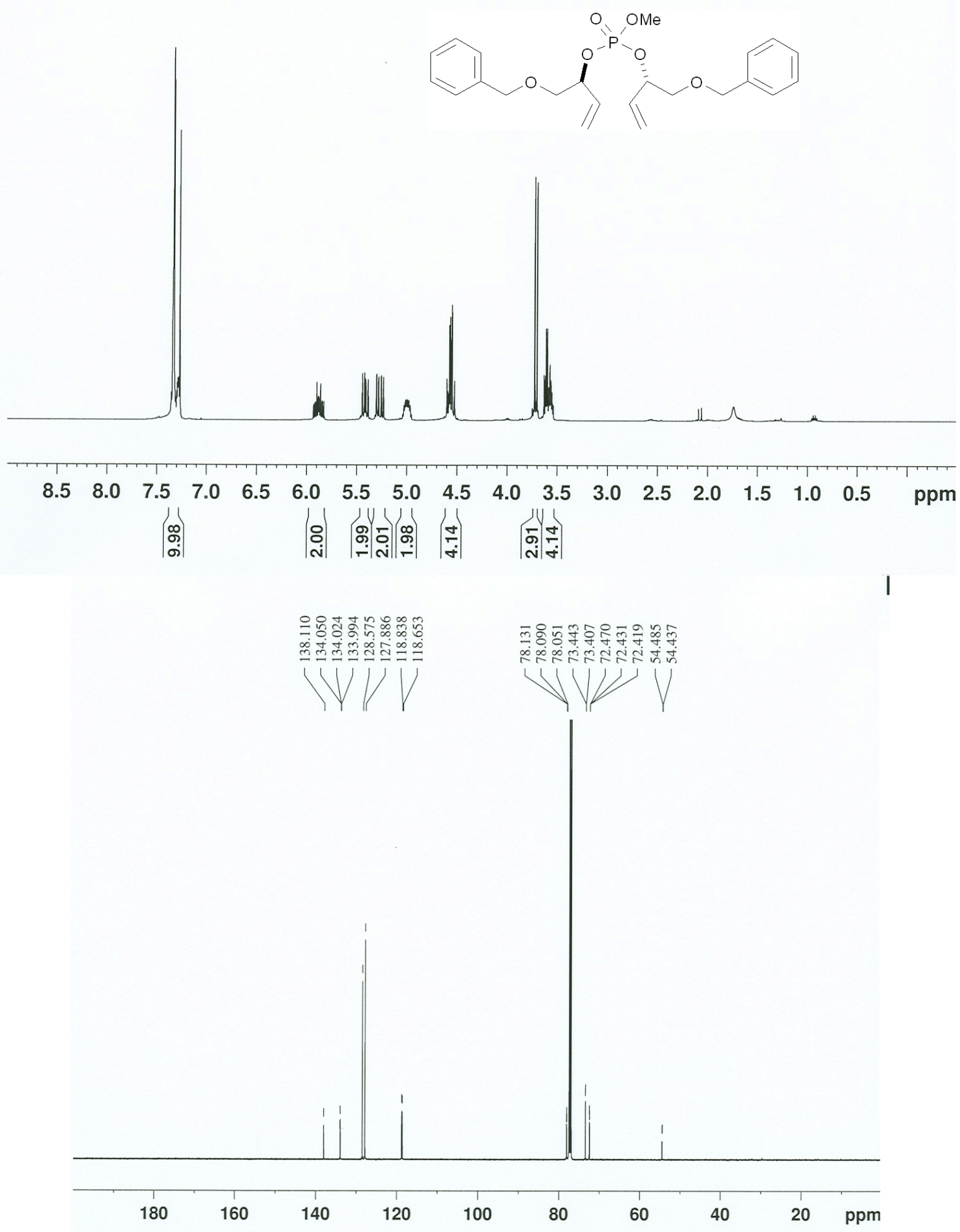




\section{(S,S)-Monocyclic phosphate- Compound 1}

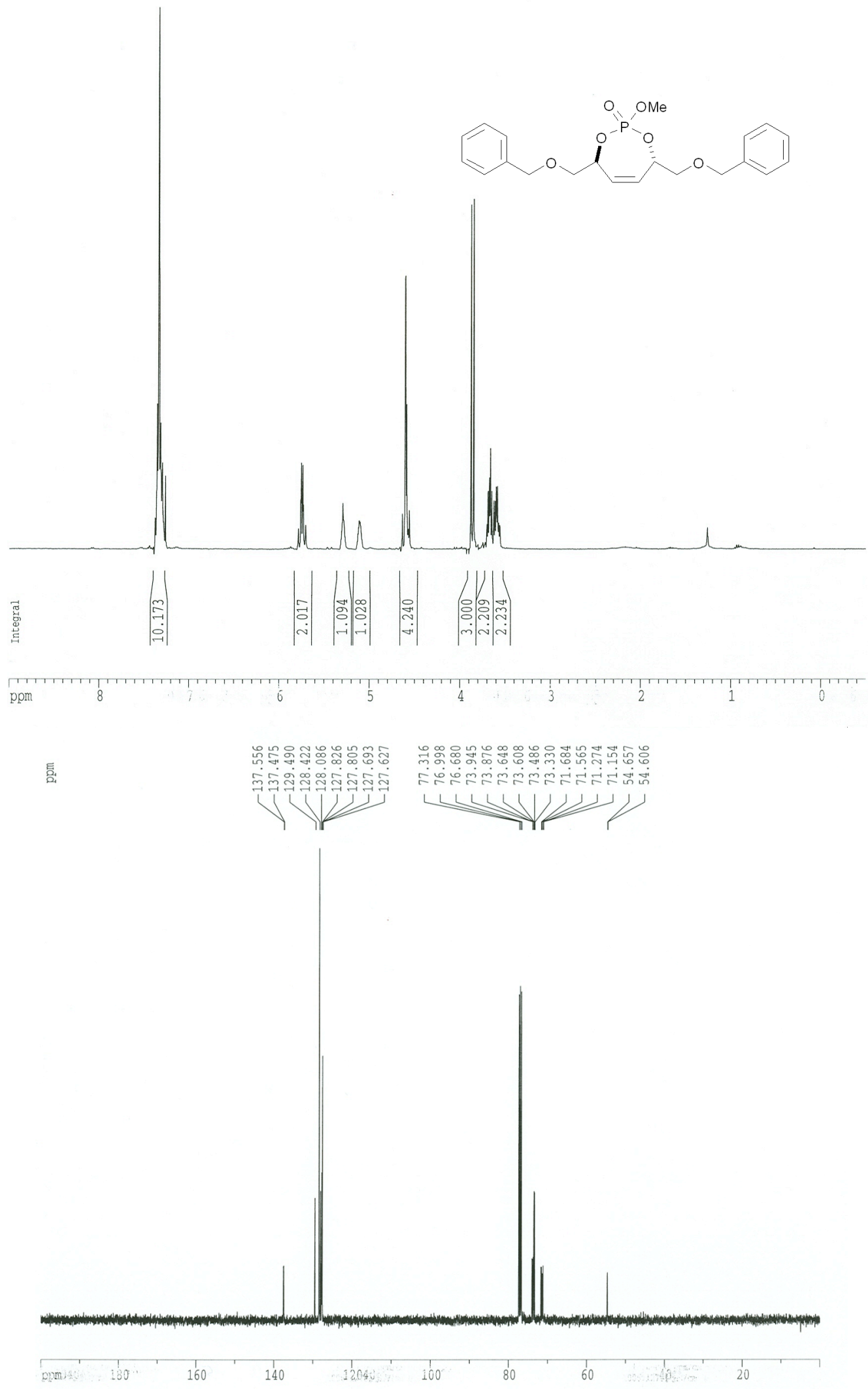



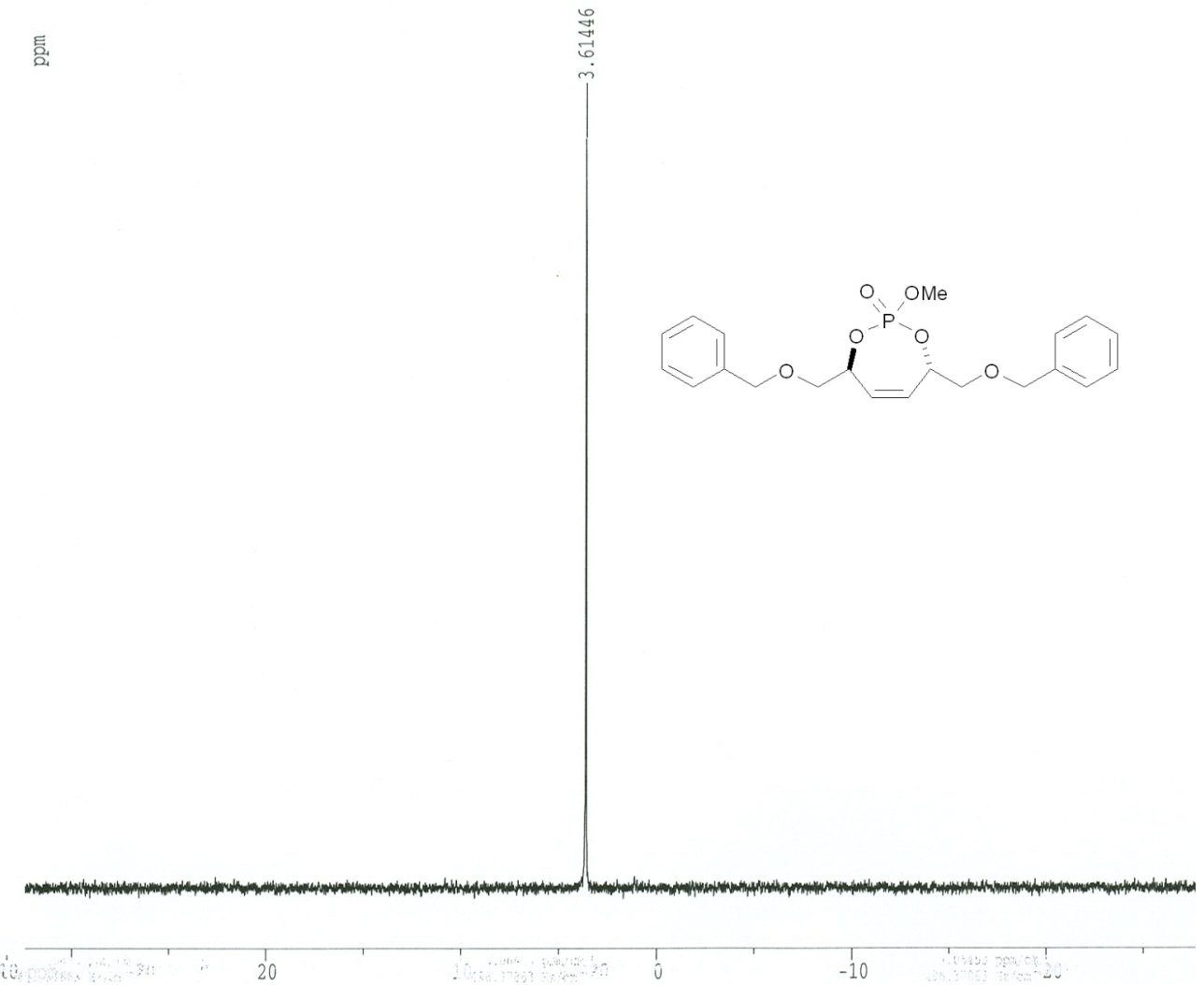
(2S,5S,Z)-1,6-bis(benzyloxy)hex-3-ene-2,5-diol- Compound 4
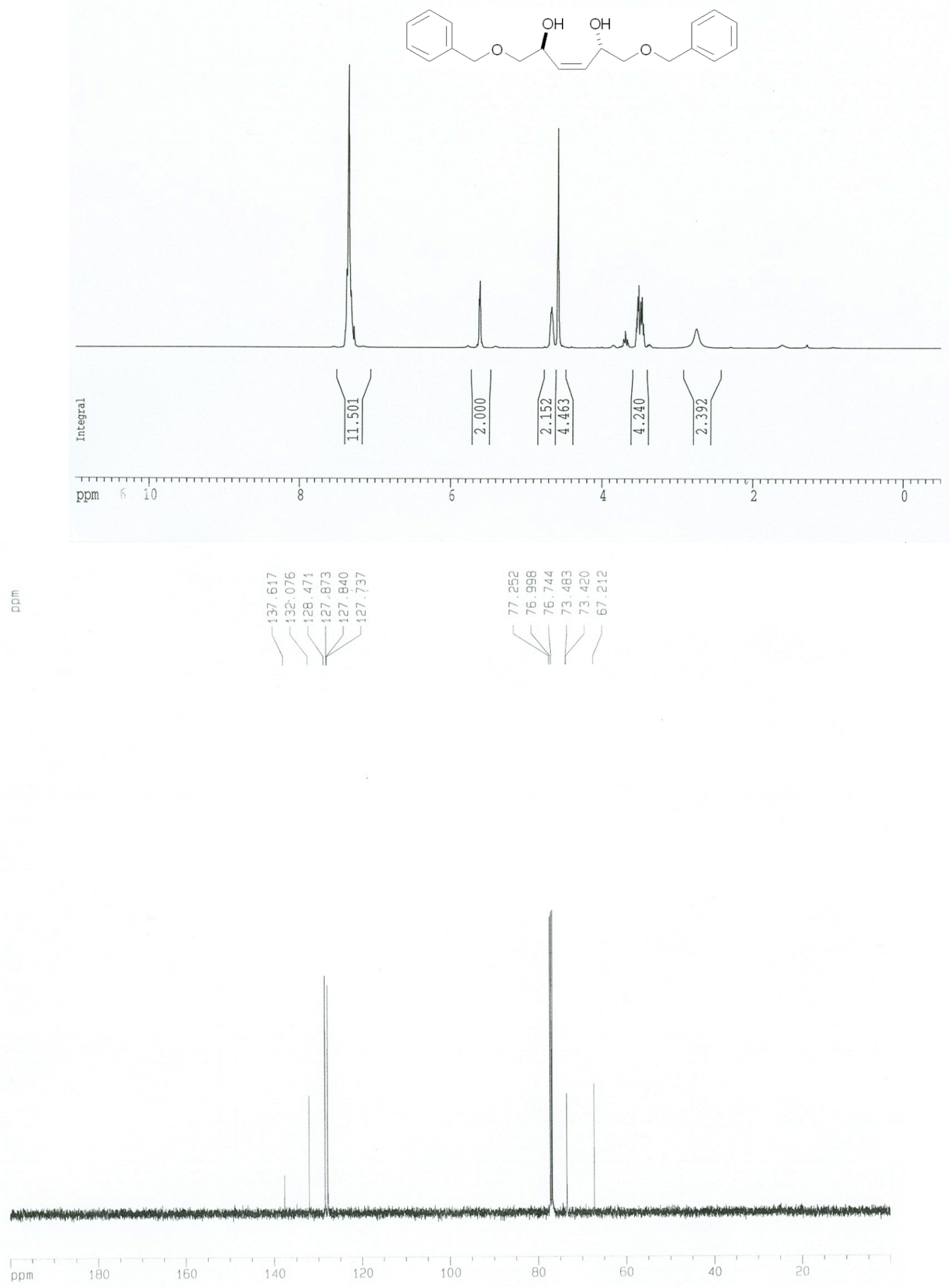
(S,S)-Hydrogenated monocyclic phosphate- Compound 5
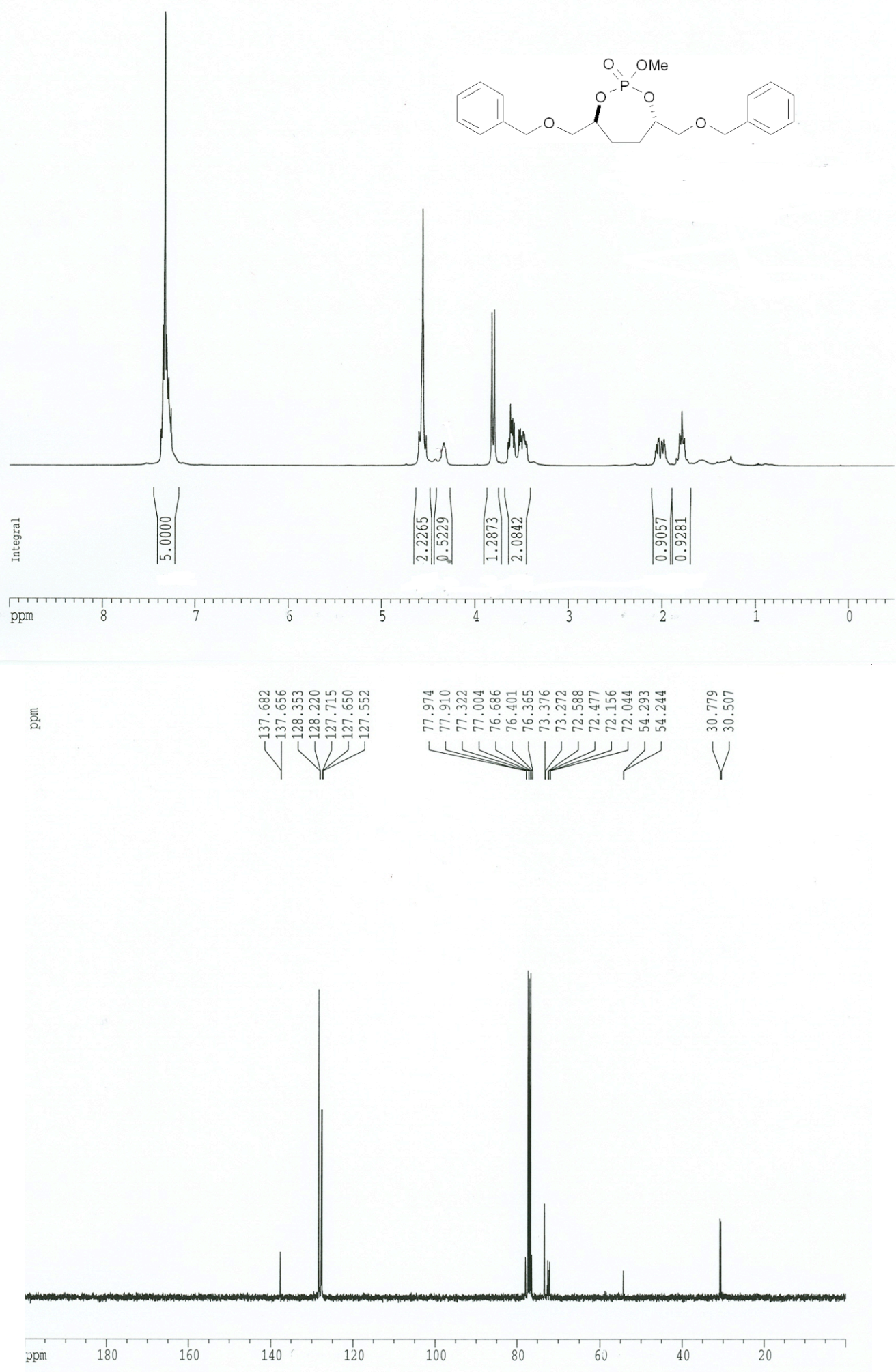


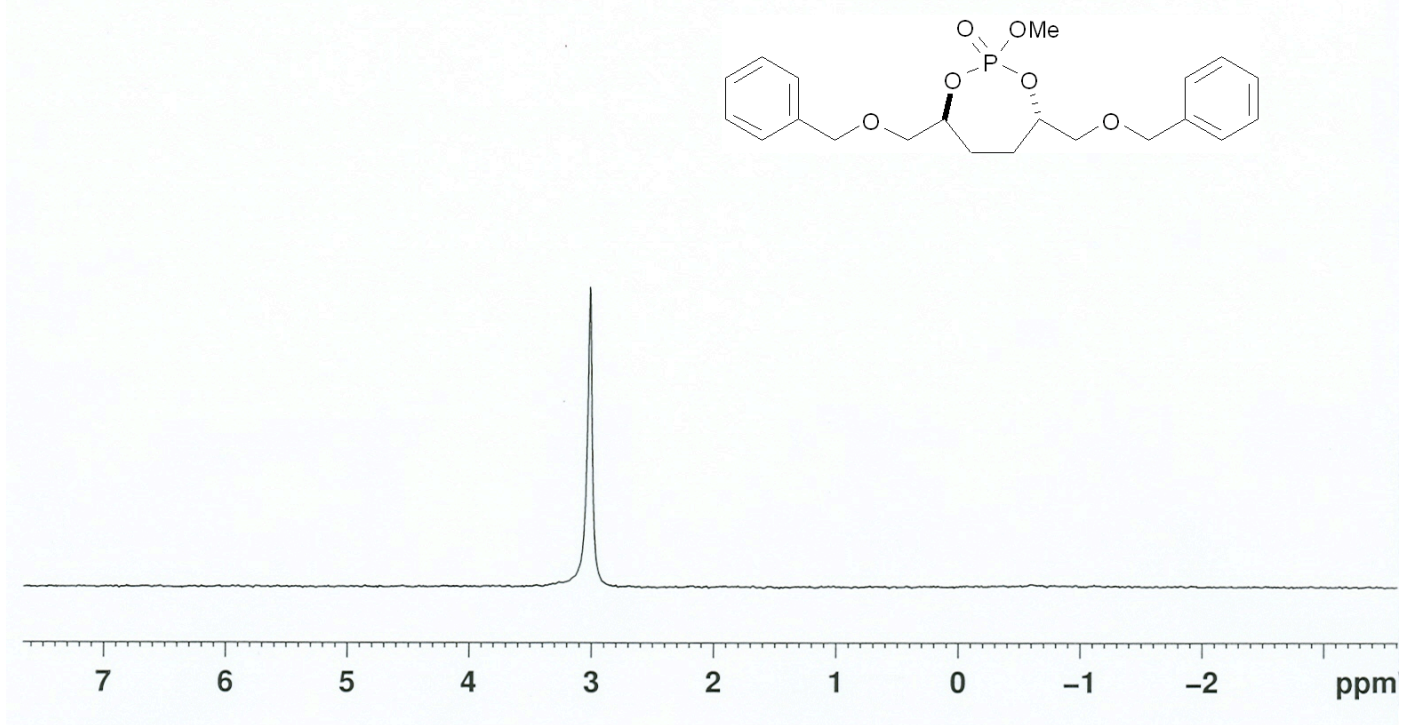


(2S,5S)-1,6-bis(benzyloxy)hexane-2,5-diol- Compound 6
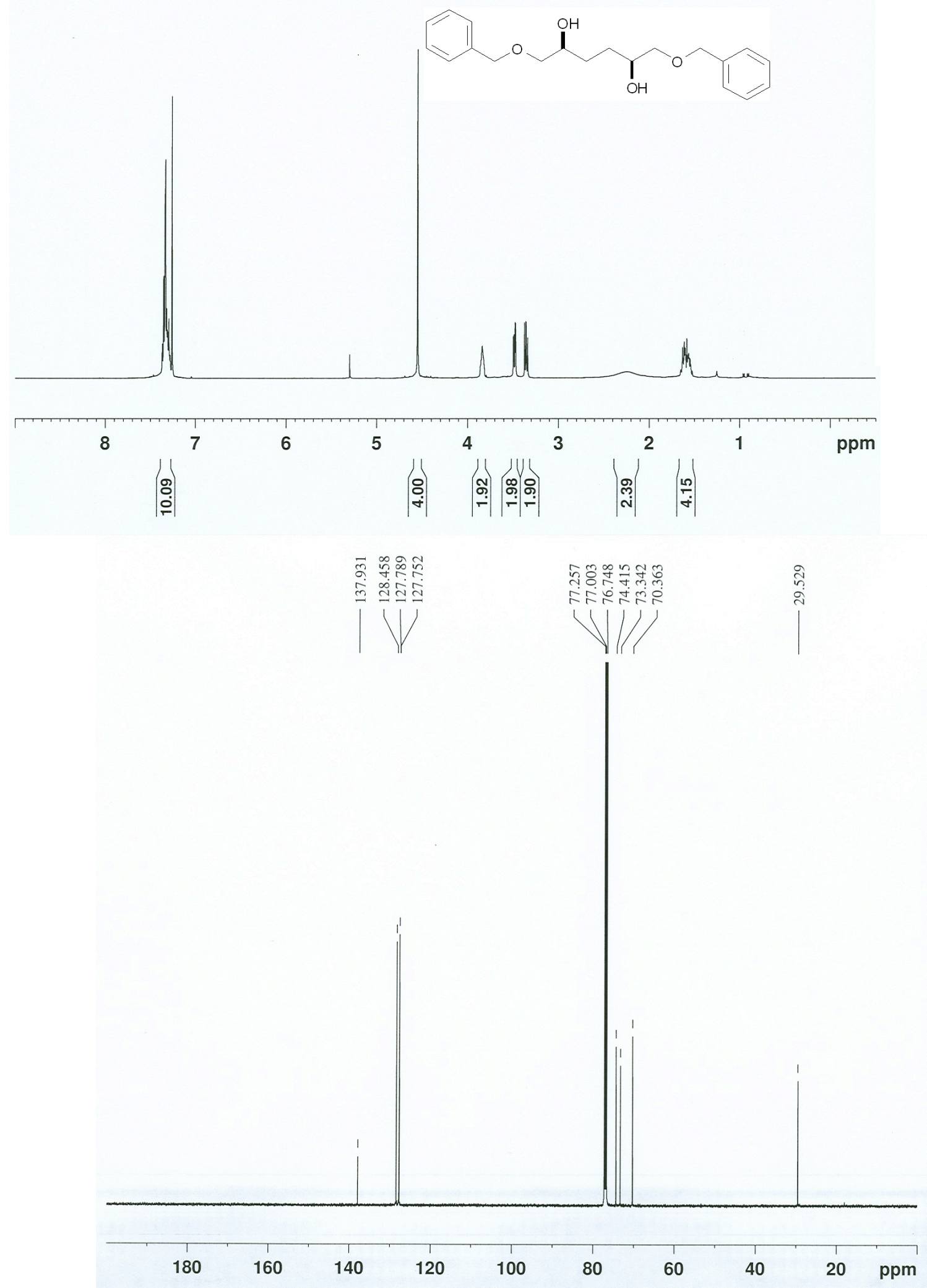
(2S,3R,E)-1,6-bis(benzyloxy)-3-methylhex-4-en-2-ol-Compound 9a
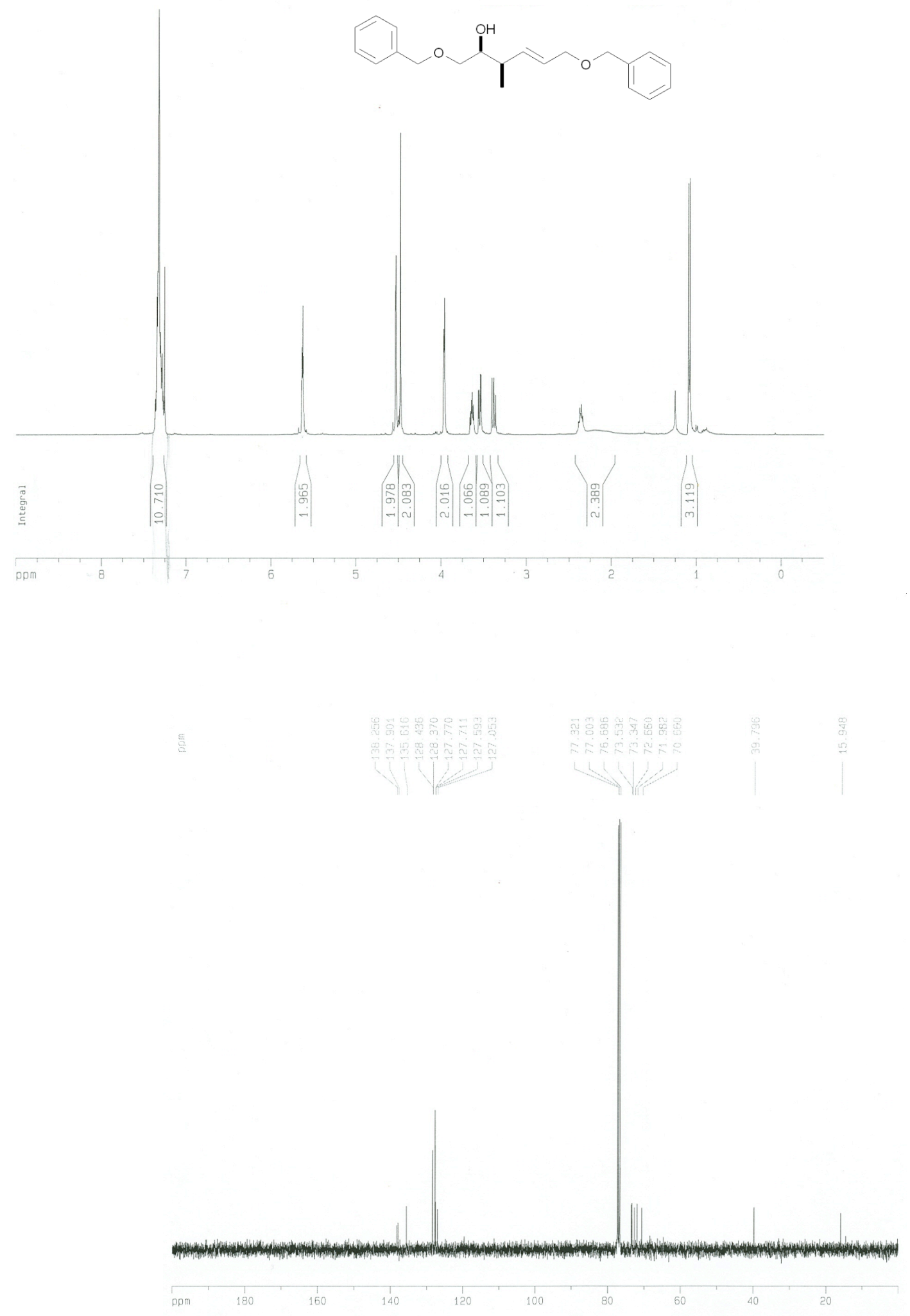

S 23 
(2S,3R,E)-1,6-bis(benzyloxy)-3-ethylhex-4-en-2-ol- Compound 9b
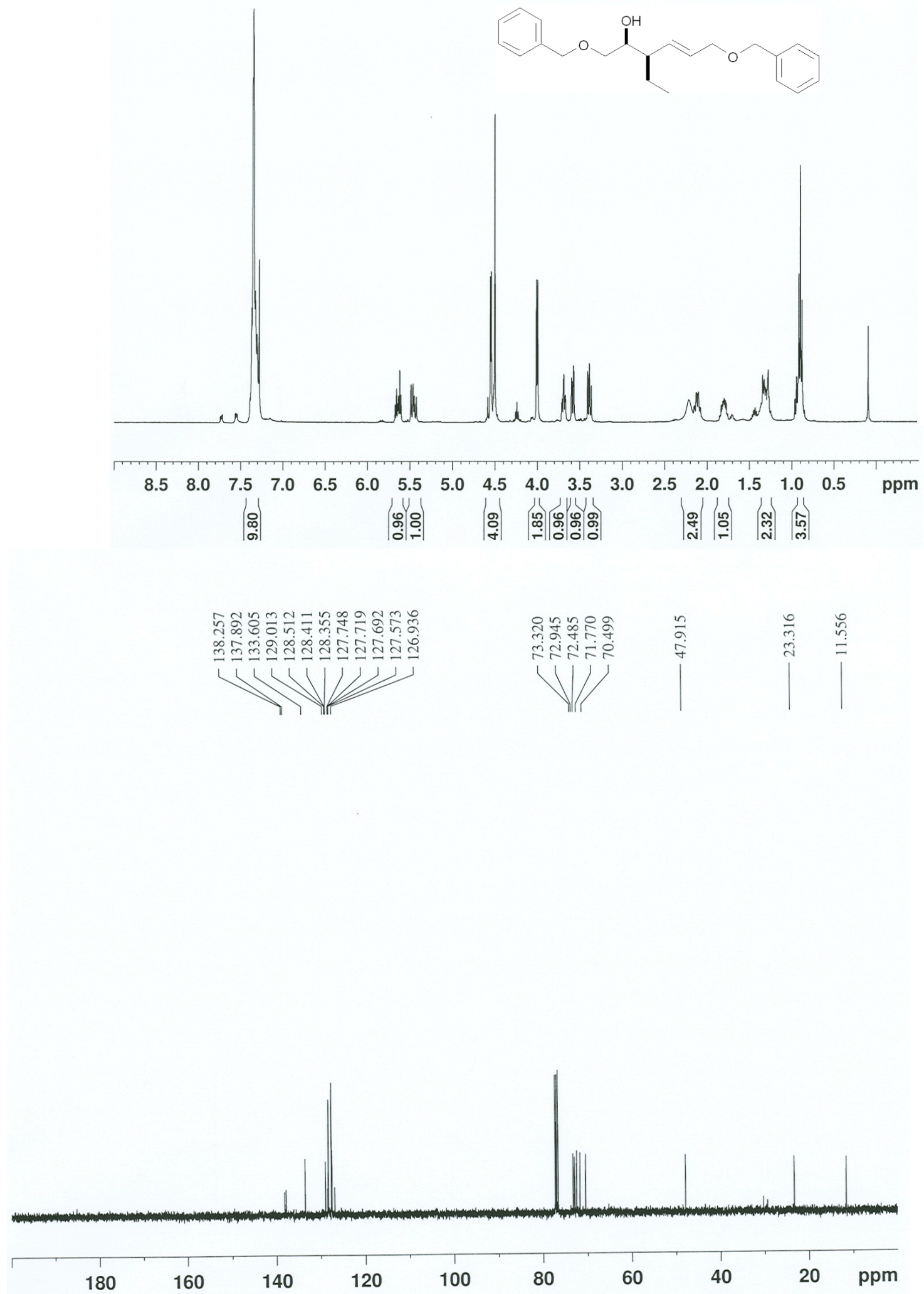
(2S,3R,E)-1,6-bis(benzyloxy)-3-isopropylhex-4-en-2-ol- Compound 9c
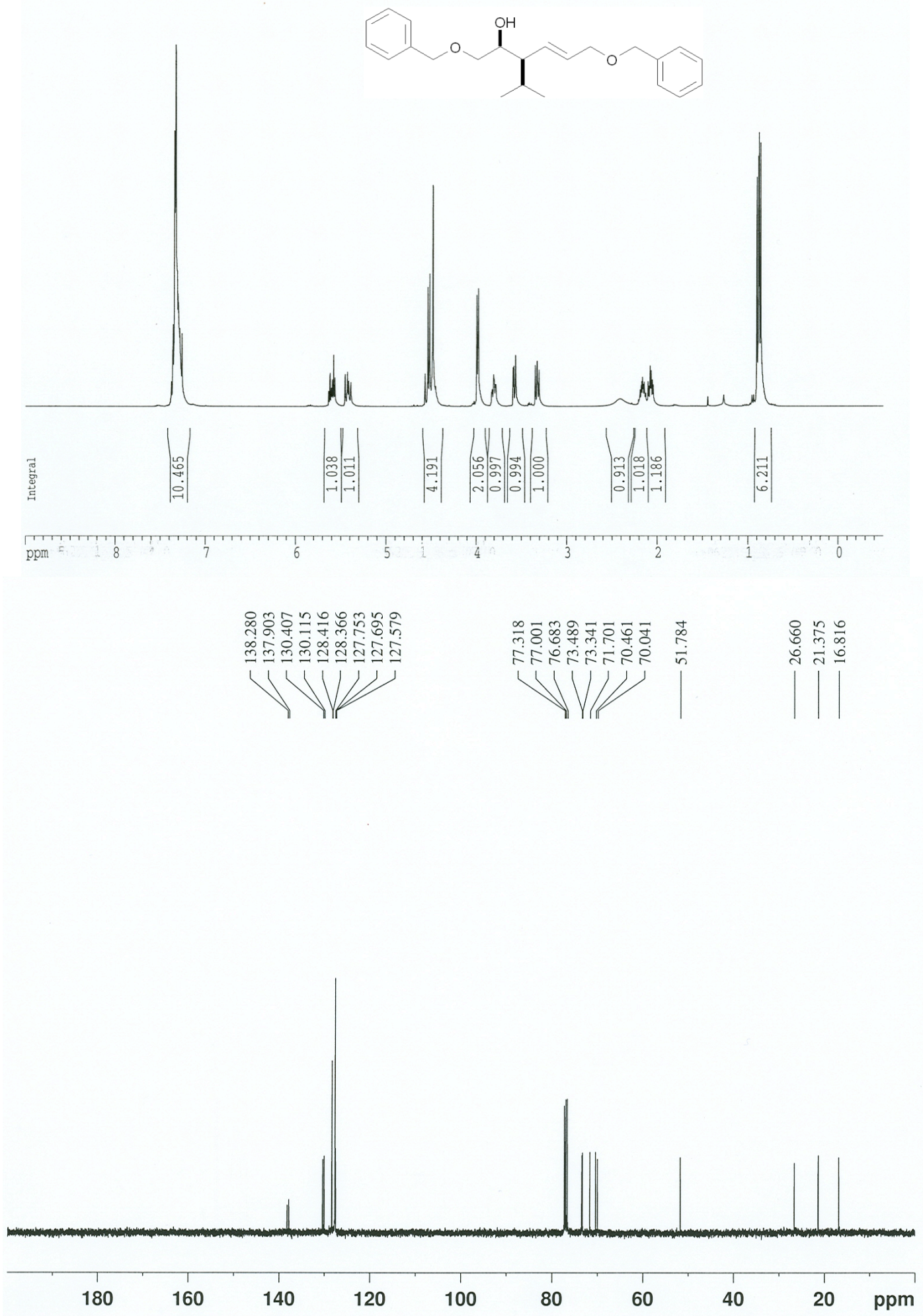


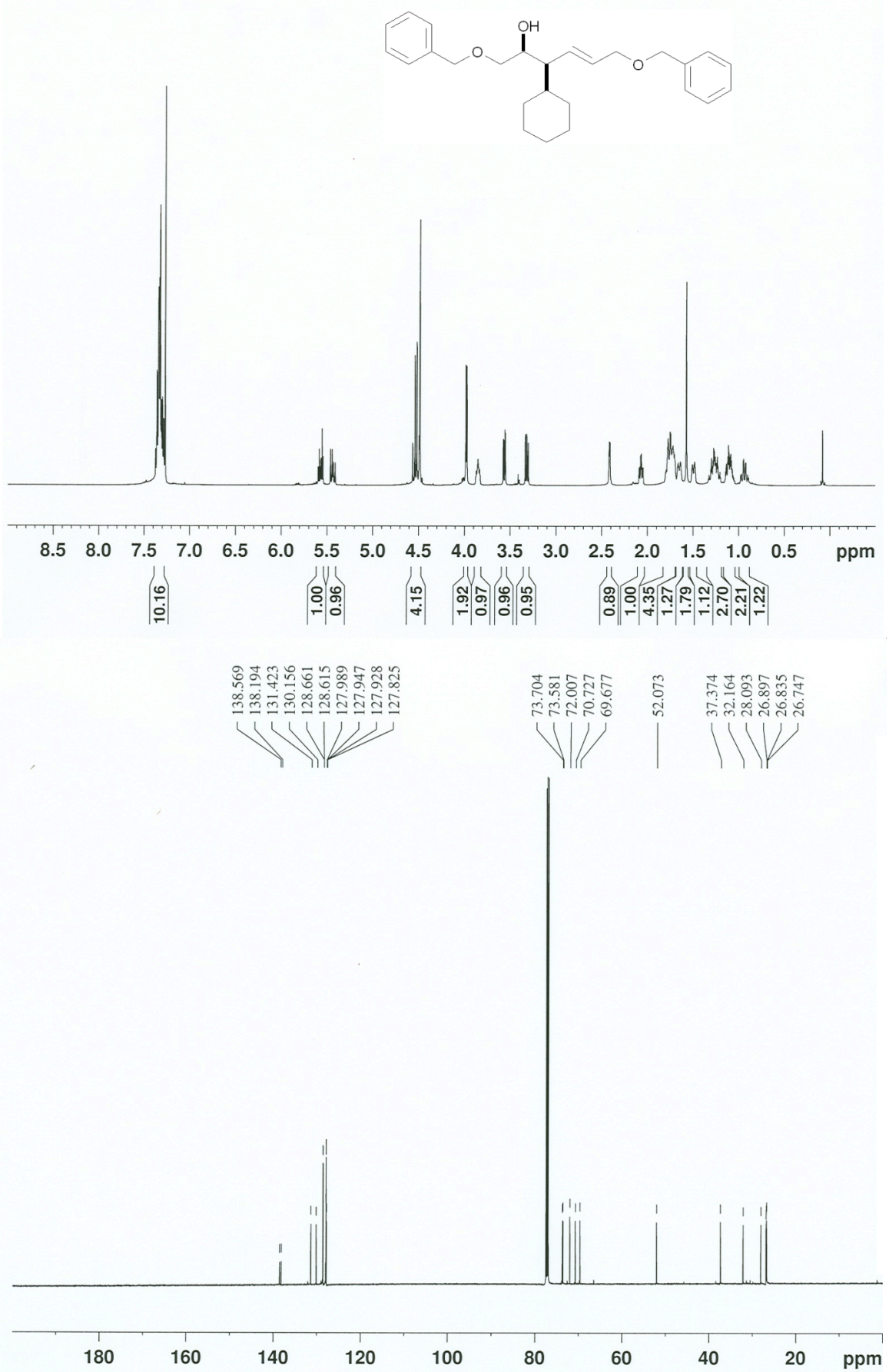




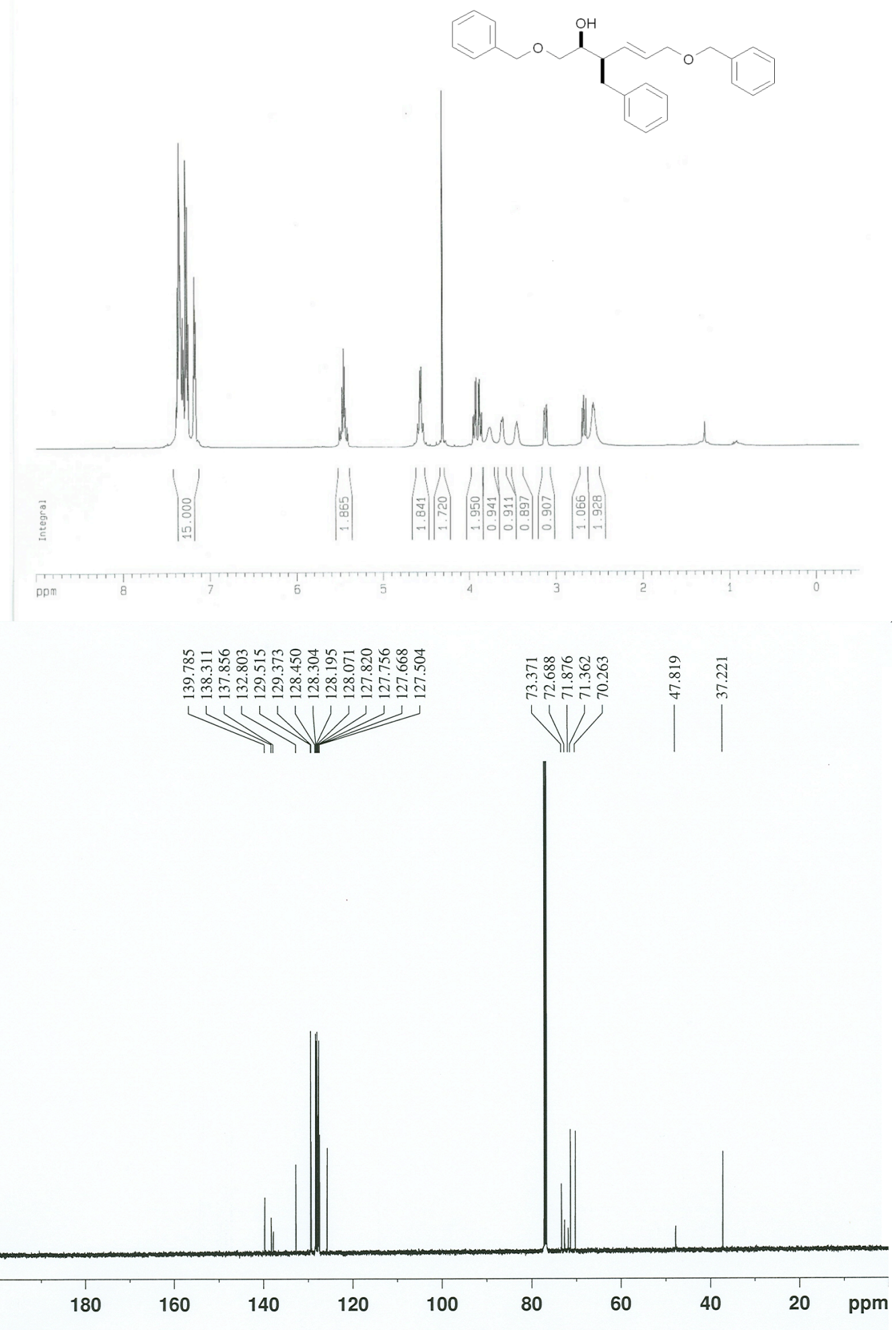


(R,E)-8-(benzyloxy)-5-((S)-2-(benzyloxy)-1-hydroxyethyl)oct-6-enenitrile- Compound 9f
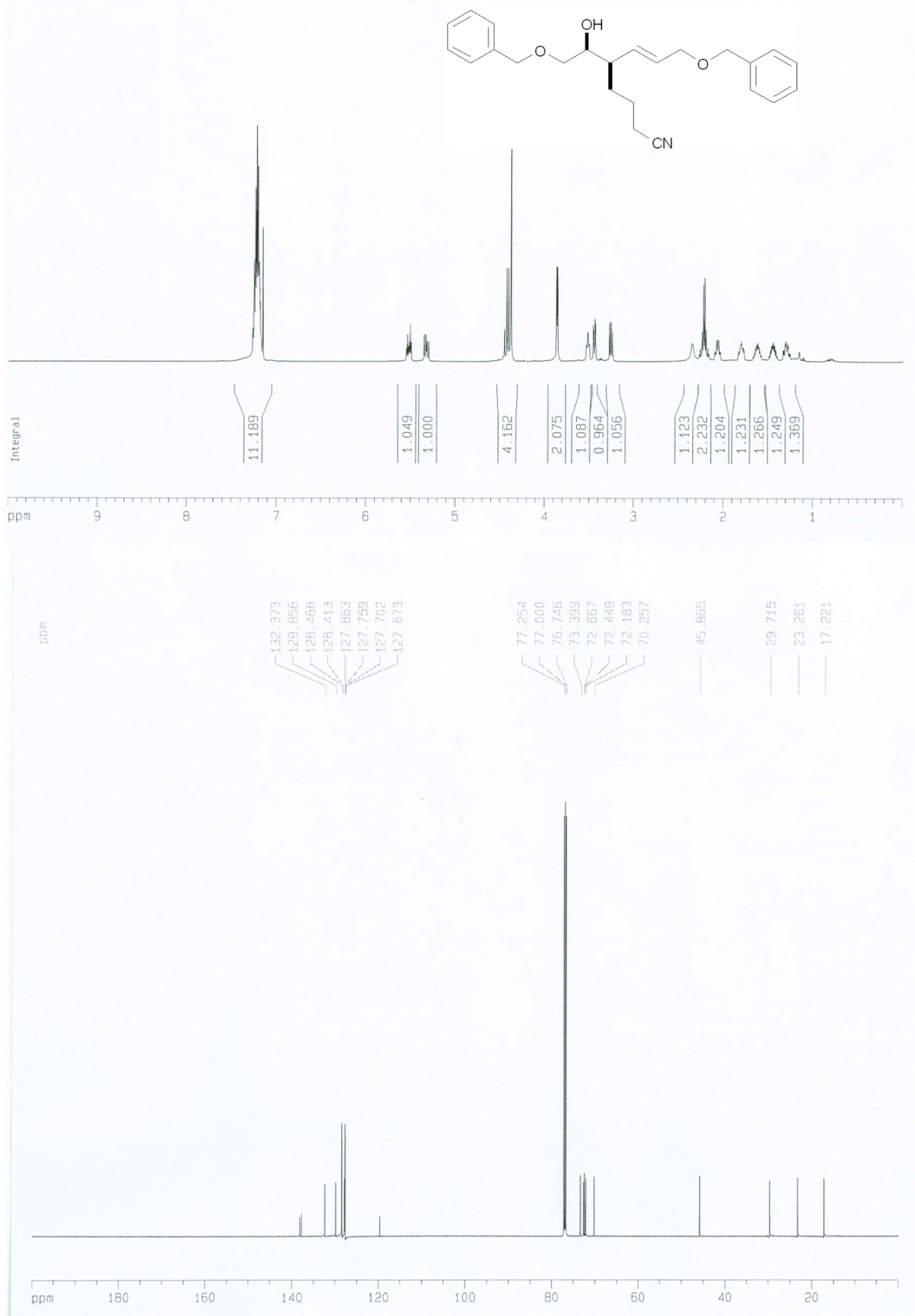
(2S,3R)-1-(benzyloxy)-3-((E)-3-(benzyloxy)prop-1-enyl)-7-chloroheptan-2-ol- Compound 9g
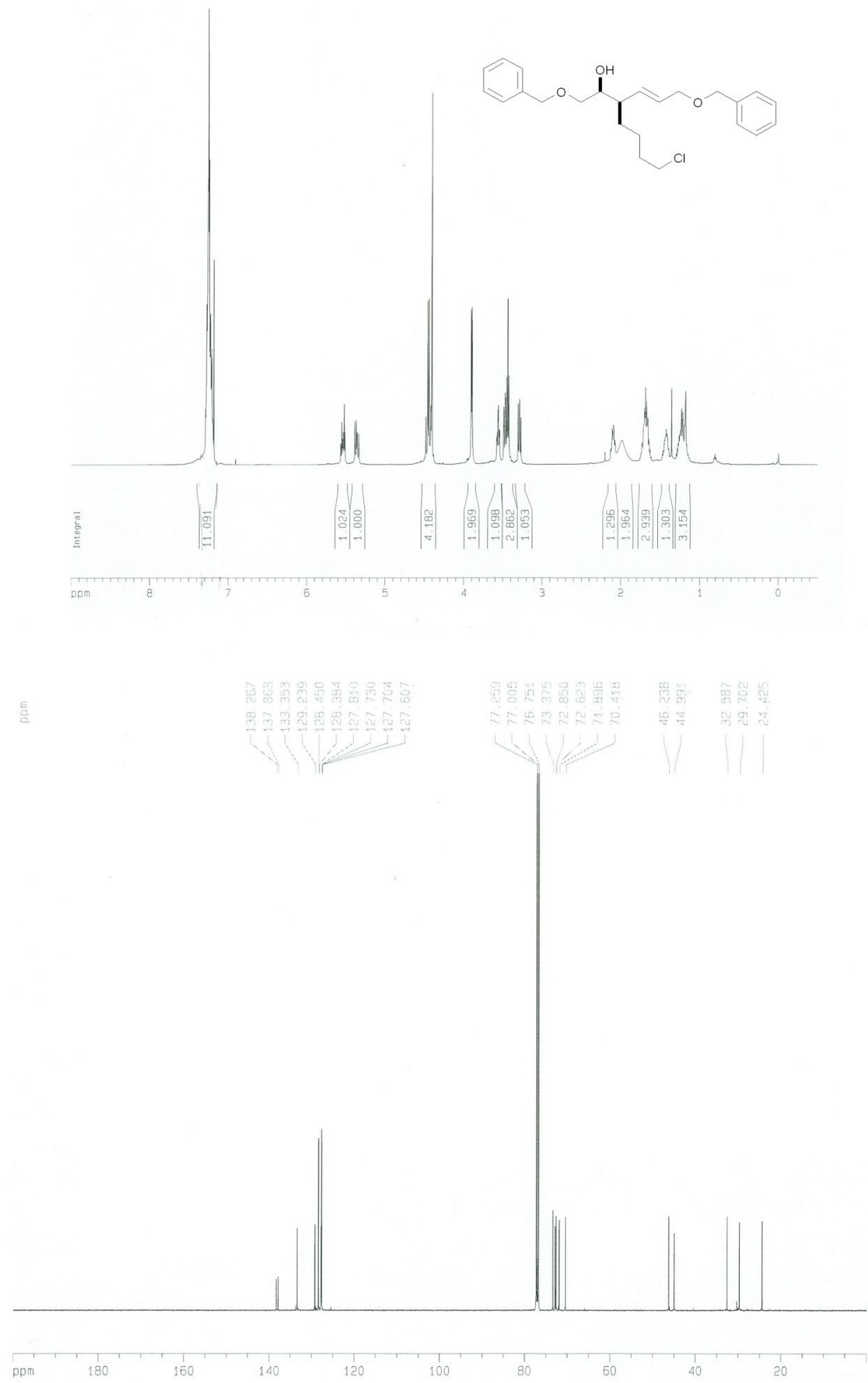
(2S,3R)-1-(benzyloxy)-3-((E)-3-(benzyloxy)prop-1-enyl)oct-7-en-2-ol- Compound 9h
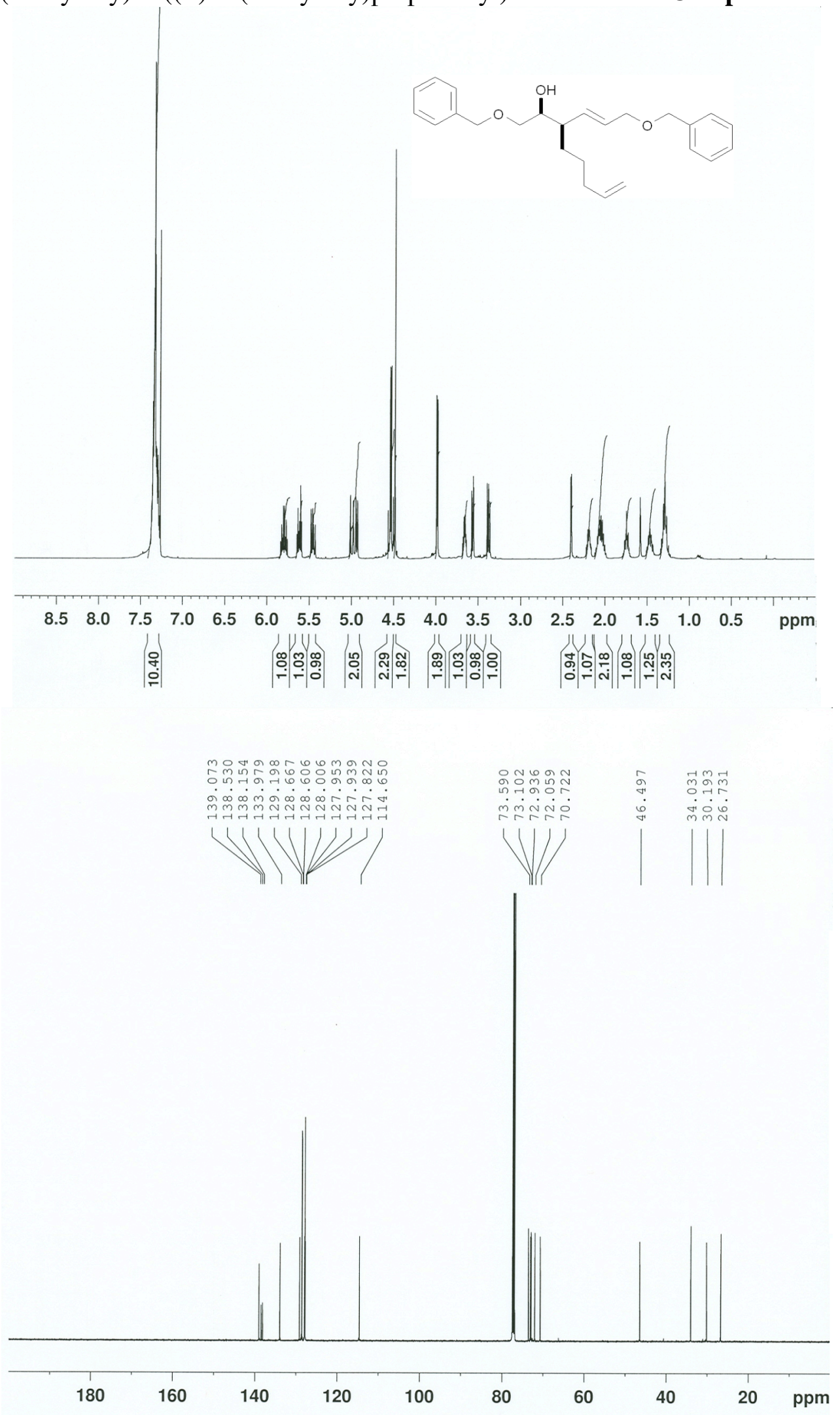
(2S,3R,E)-1,6-bis(benzyloxy)-3-methylhex-4-en-2-yl methyl hydrogen phosphate-Compound 8a

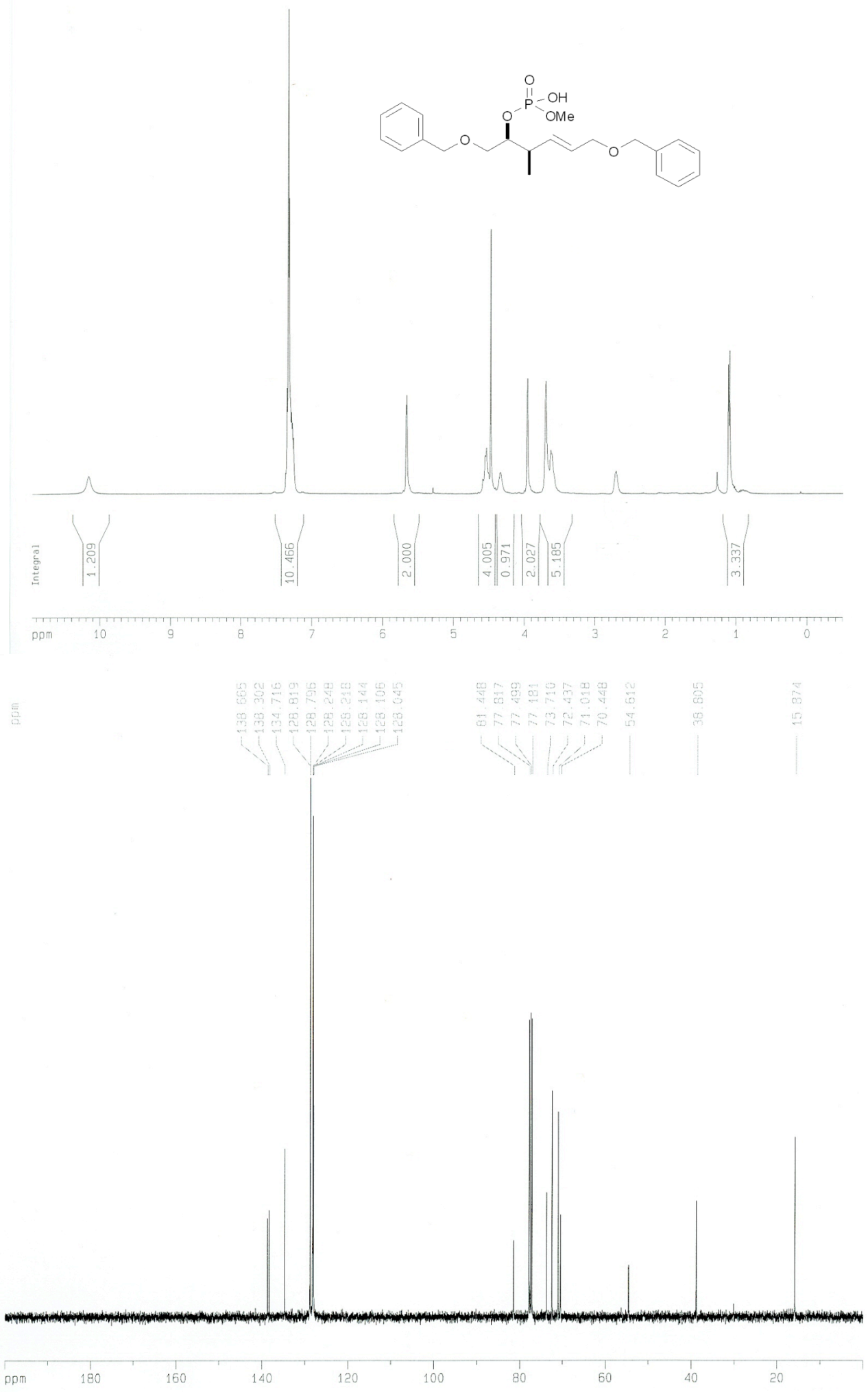




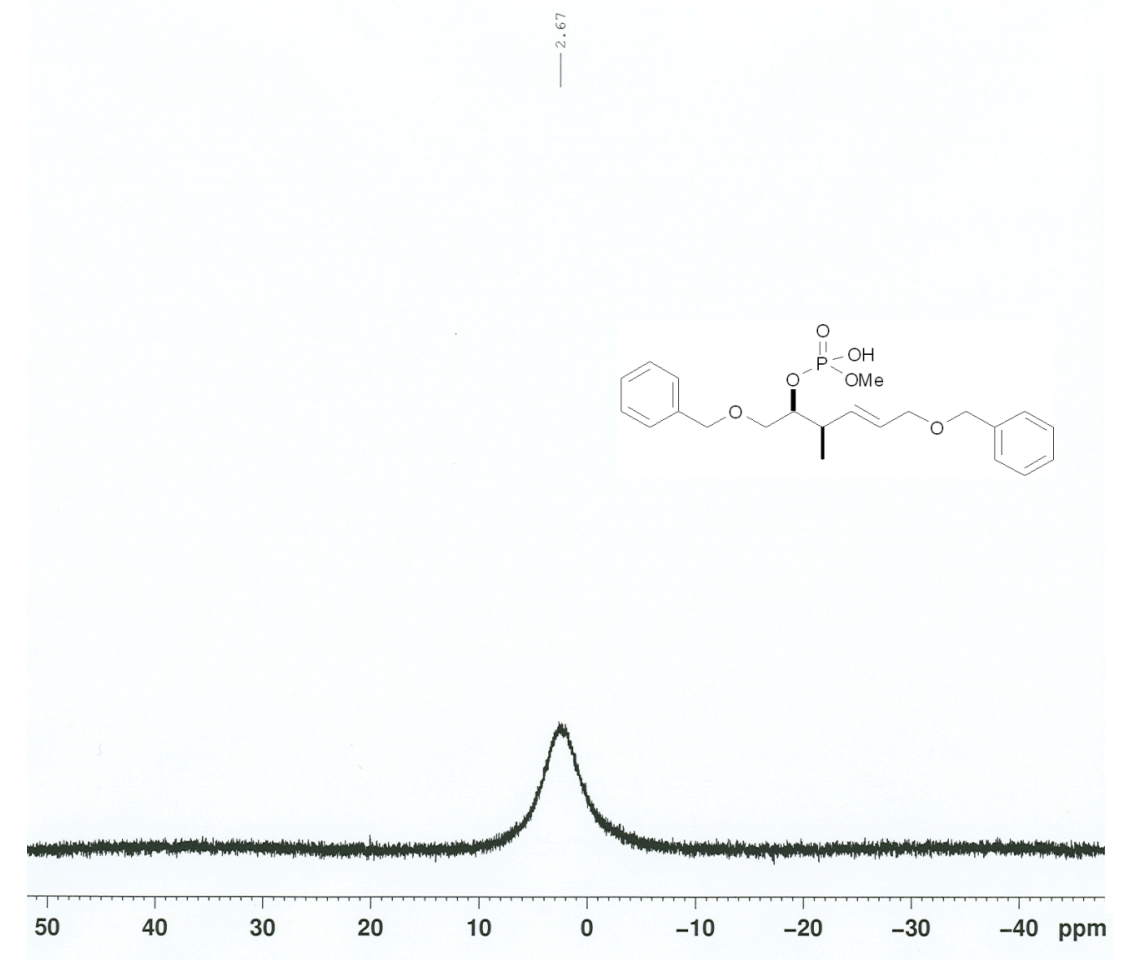

S 32 
(2S,3R,E)-1,6-bis(benzyloxy)-3-methylhex-4-en-2-yl dimethyl phosphate-Compound 10a
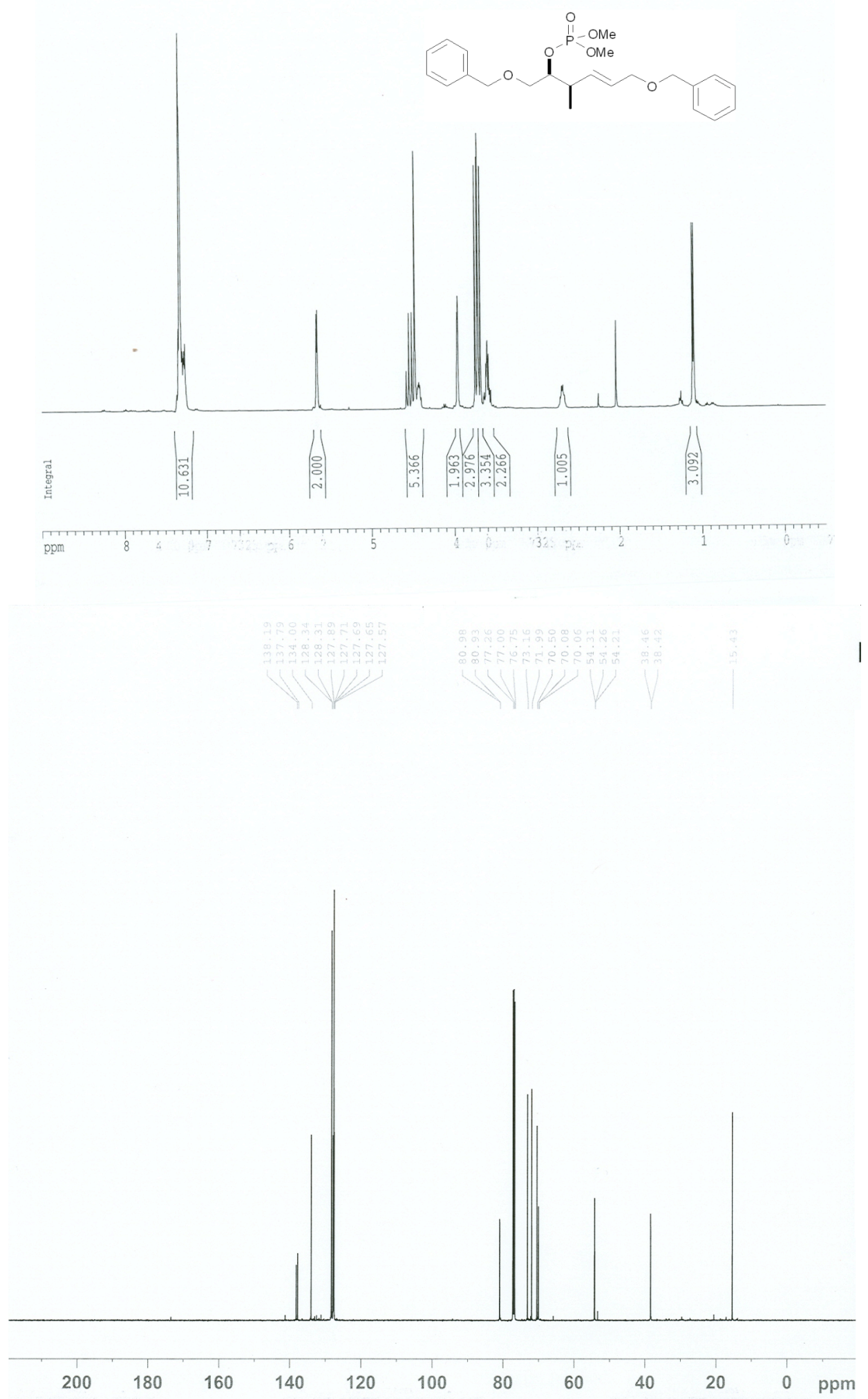

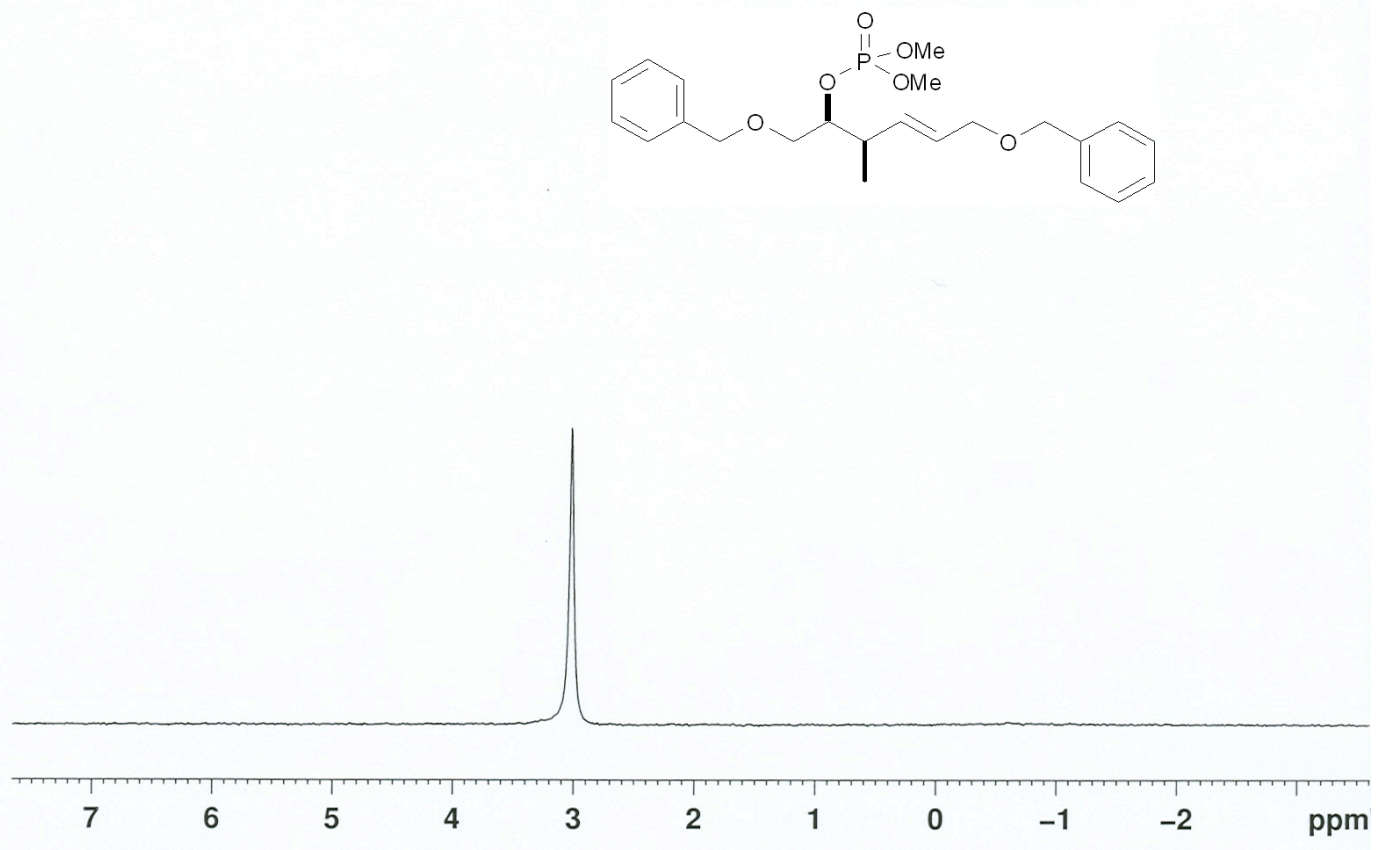
Unsymmetric Monophosphate- Compound 11
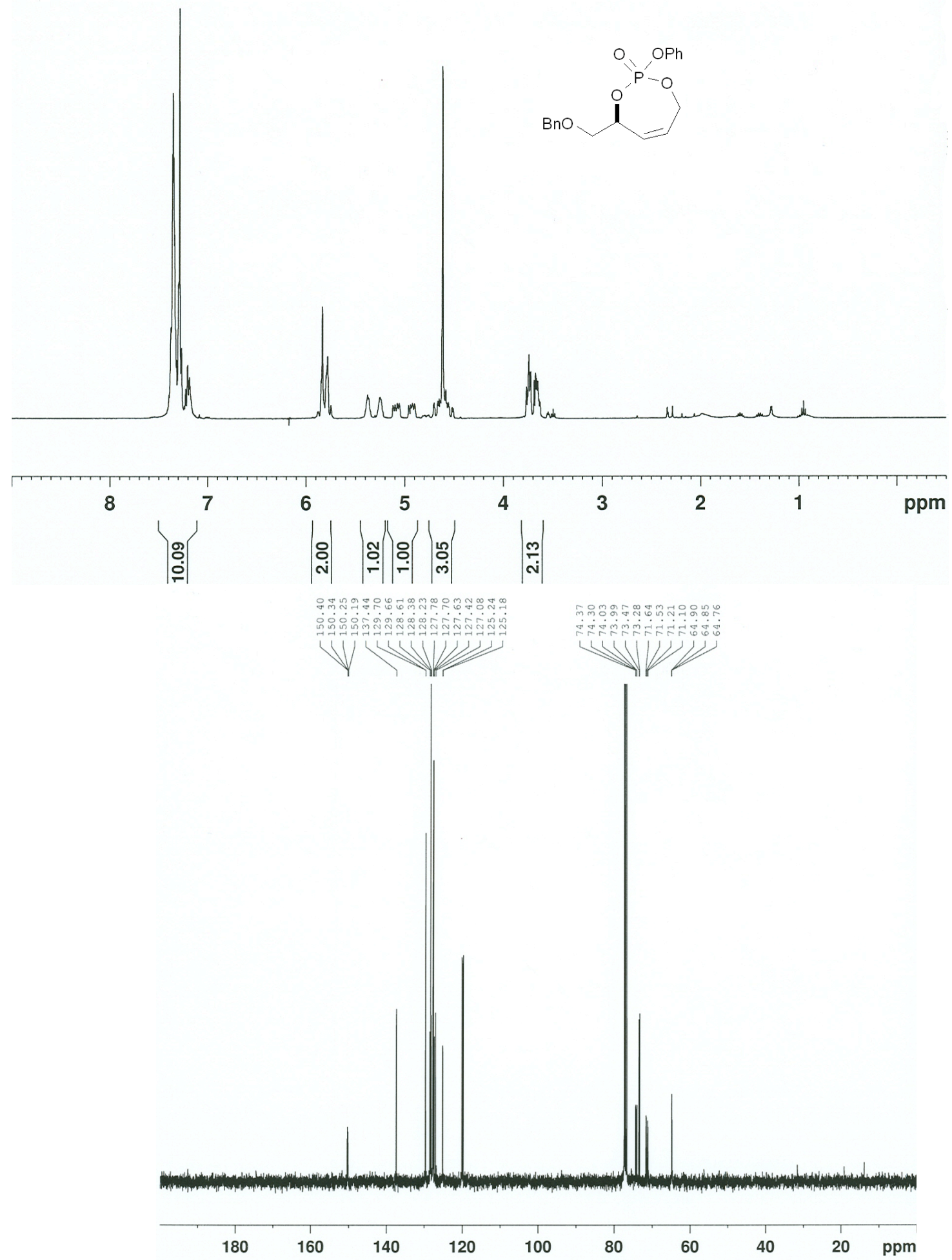


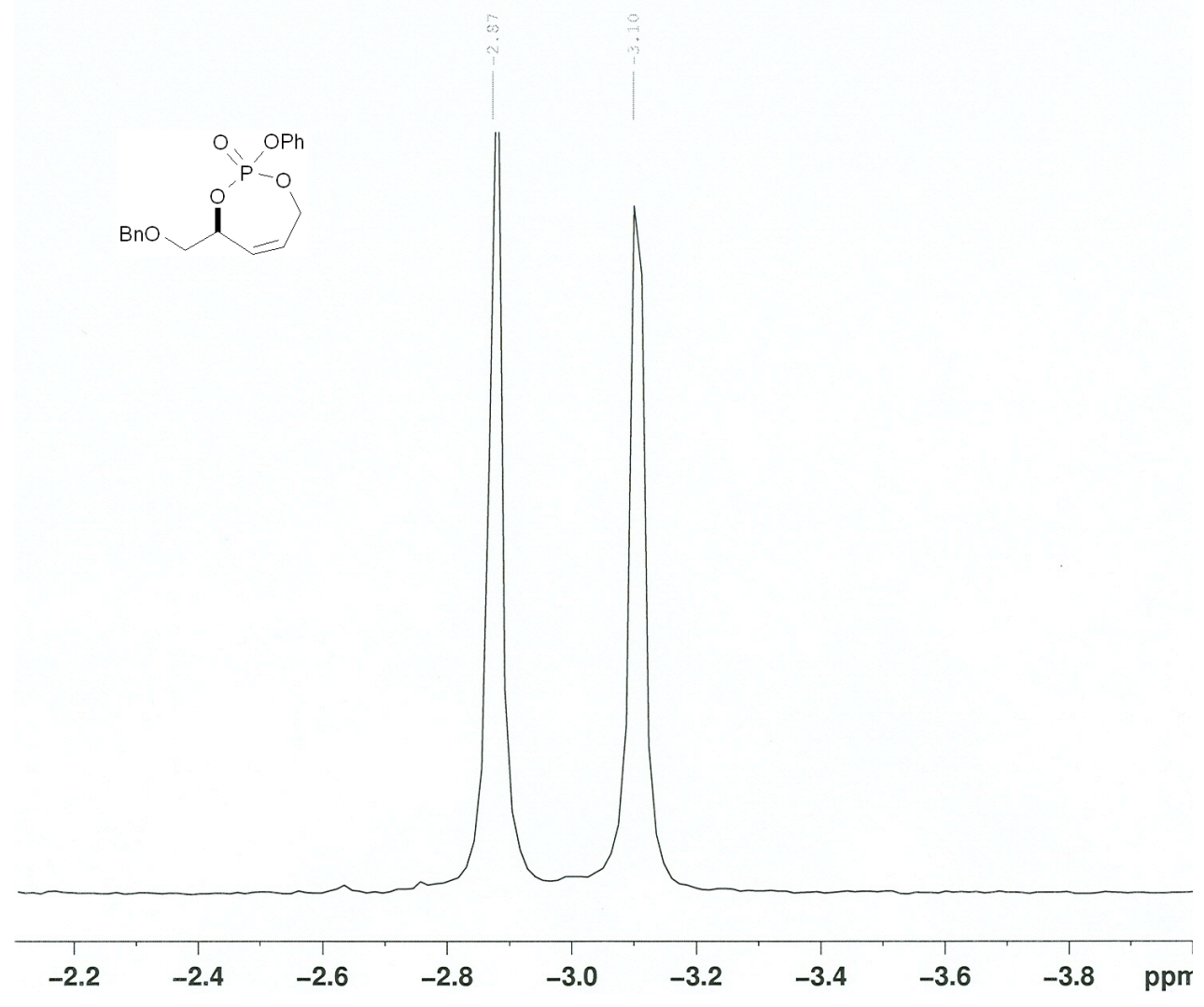


(R,E)-5-(benzyloxy)-2-ethylpent-3-en-1-ol- Compound 12

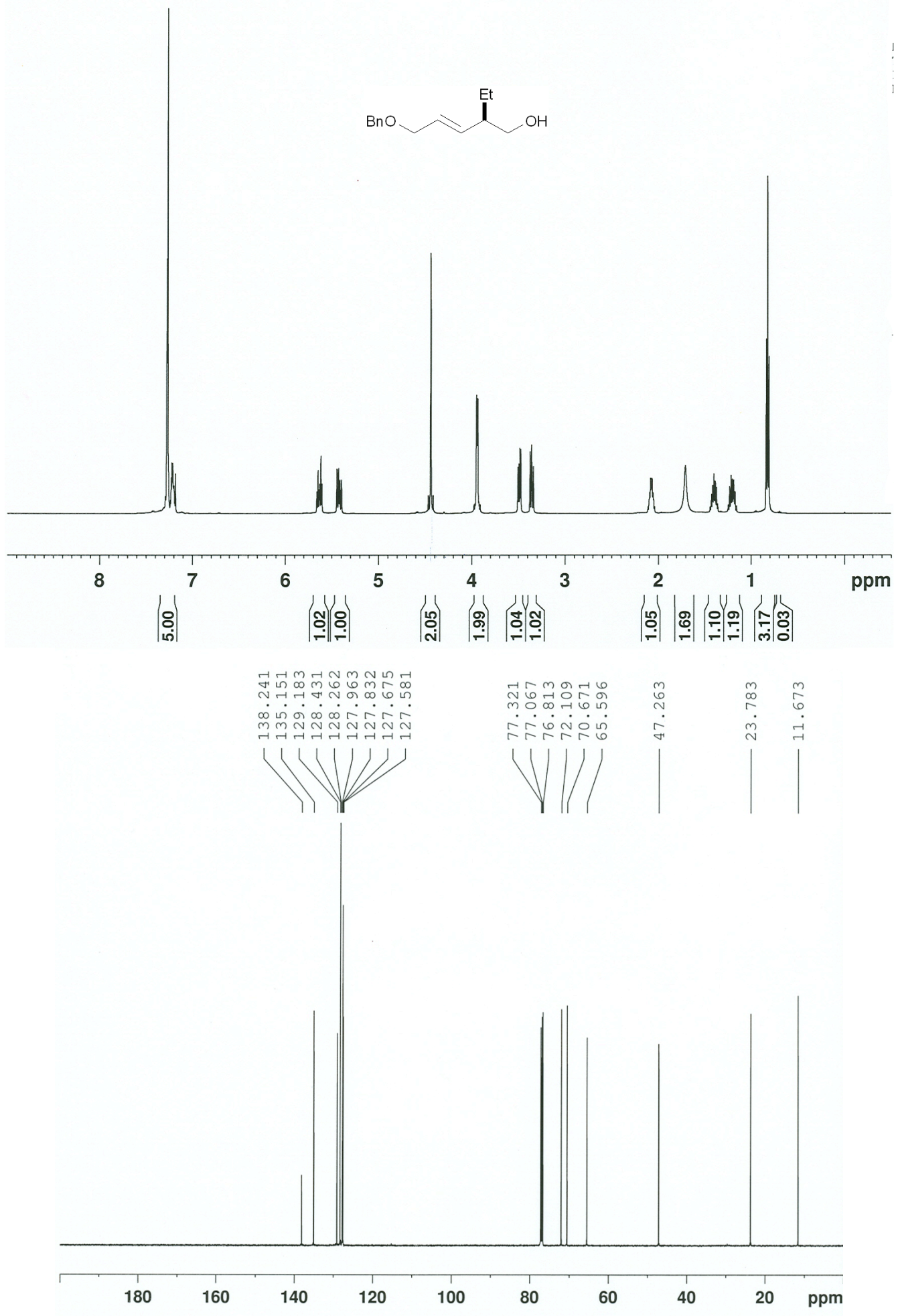


Unsymmetrical dimethyl monophosphate - Compound 14

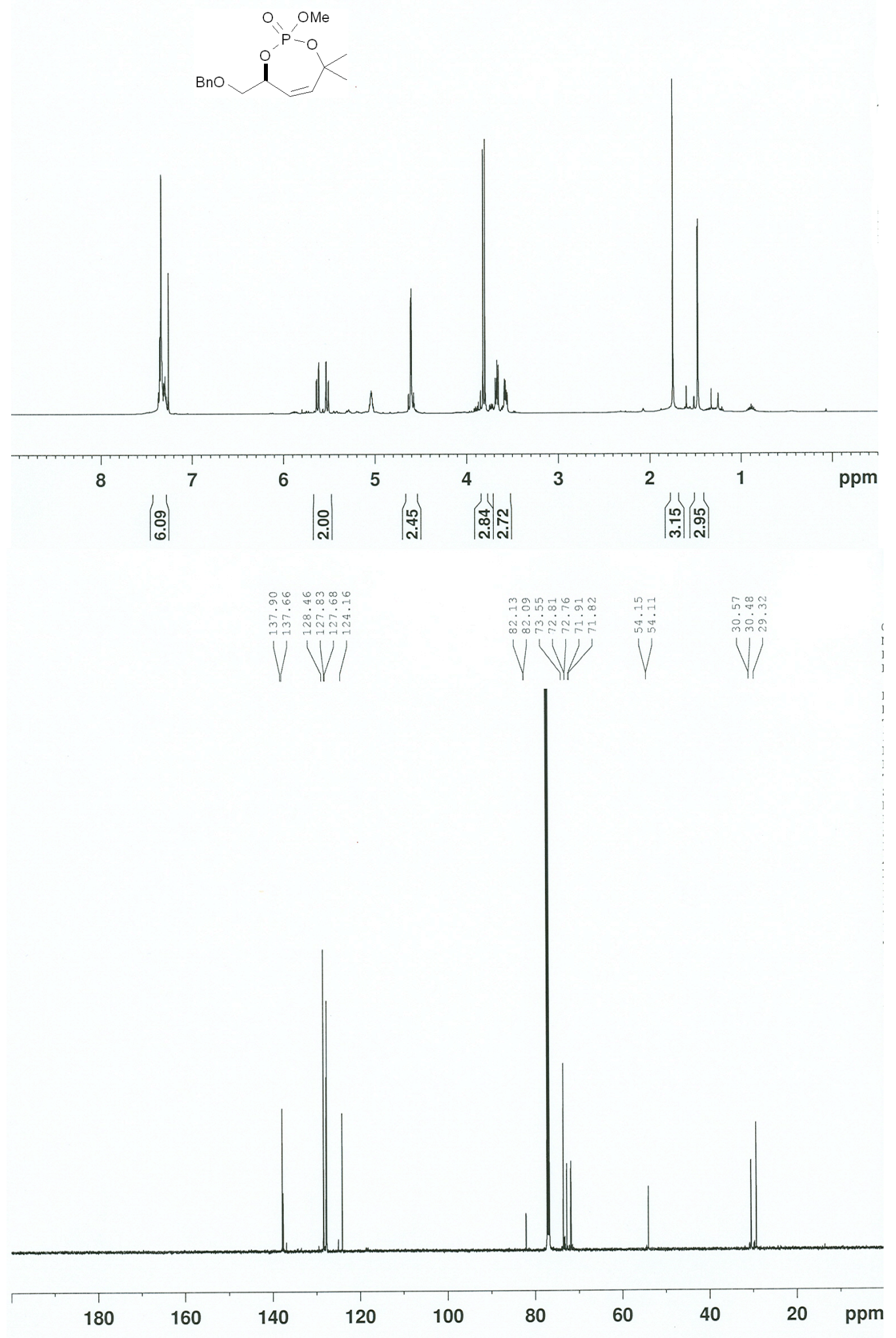




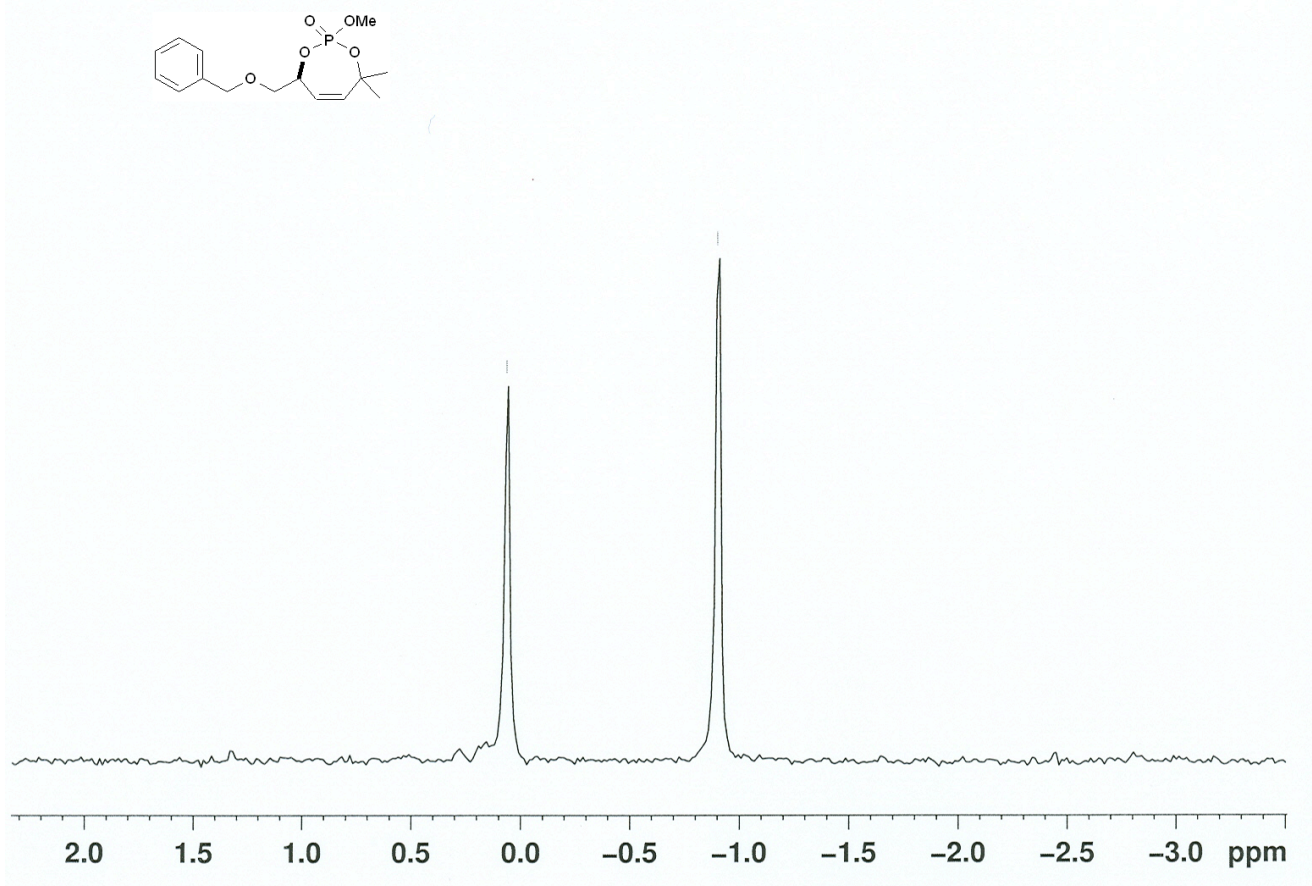


(2S,3S)-1-(benzyloxy)-3-ethyl-5-methylhex-4-en-2-ol - Compound 15
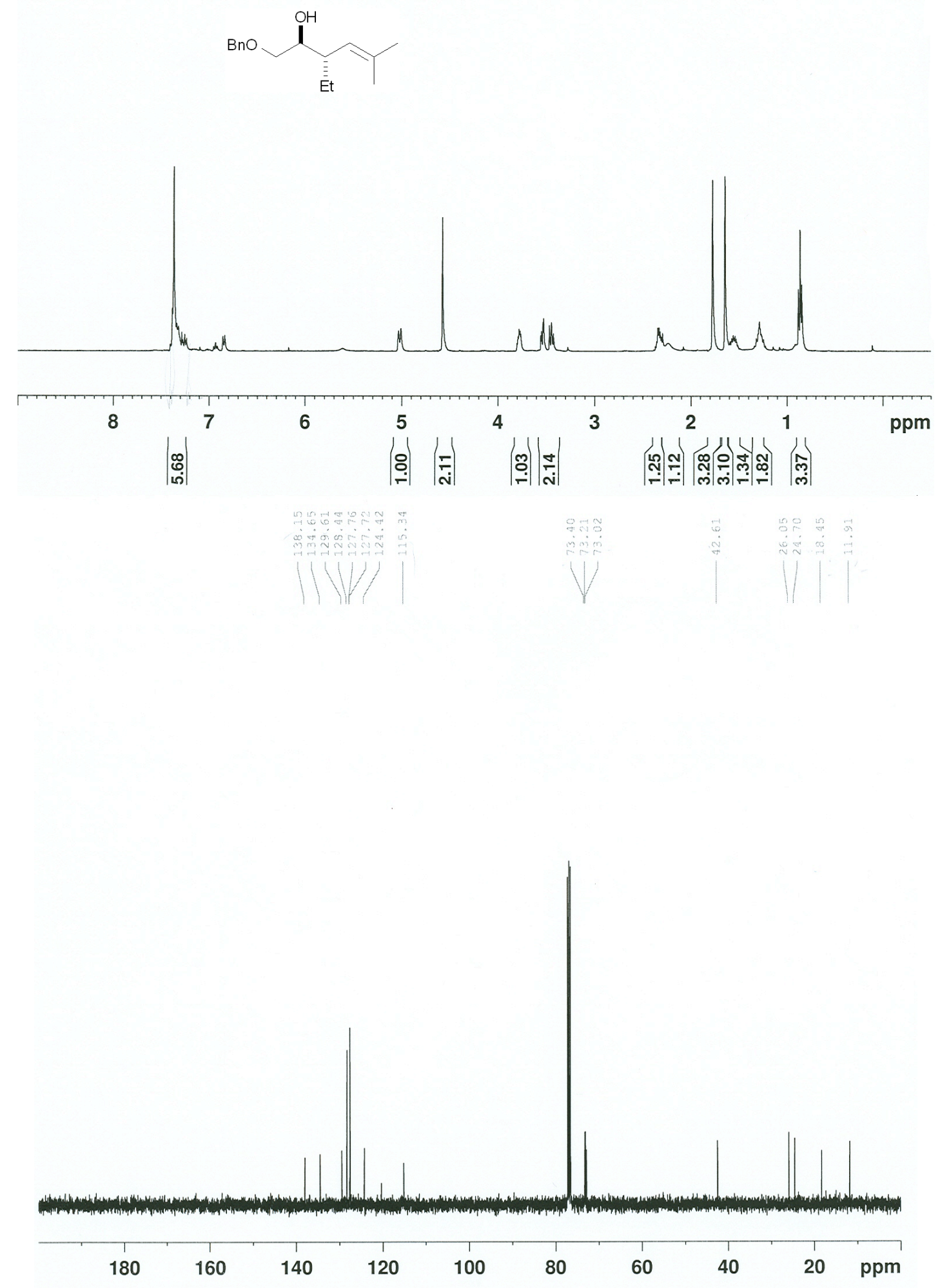
4-(benzyloxymethyl)-5-ethyl-2,2-dimethyl-1,3-dioxane

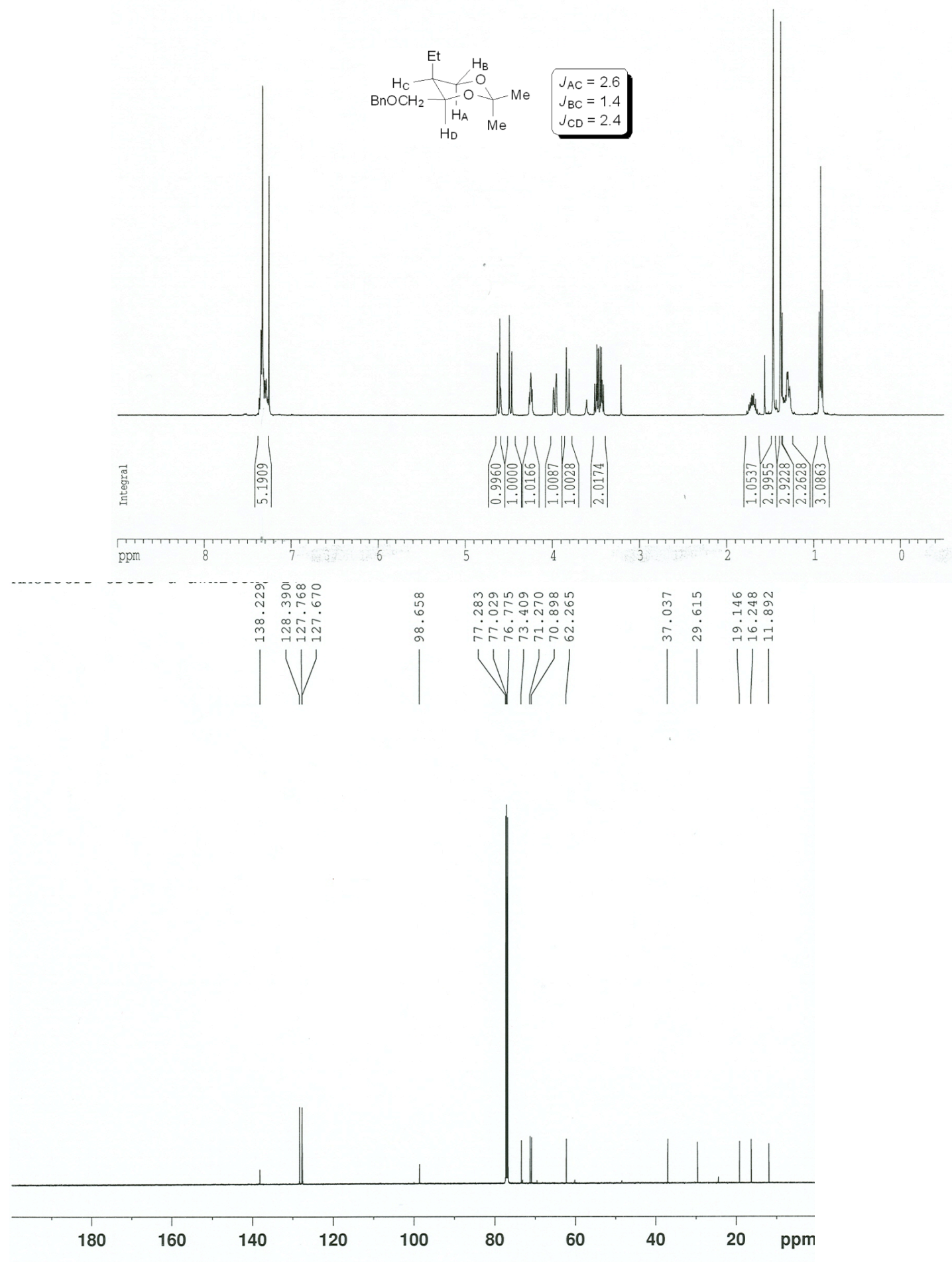


(4S,5S)-4-(benzyloxymethyl)-5-ethyl-2,2-dimethyl-1,3-dioxane
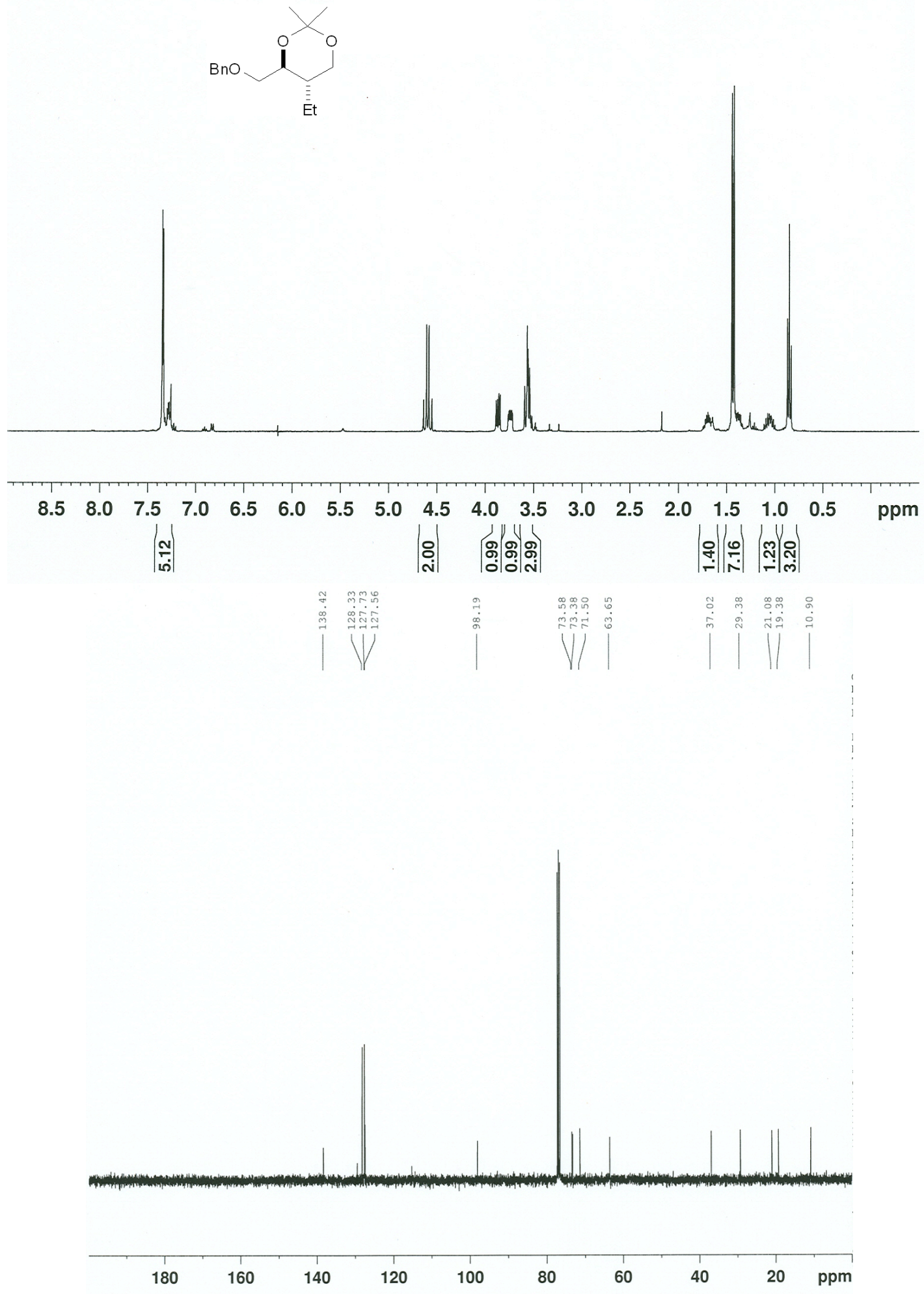